\title{
EL-IGrowCut: MRI Brain Tissue Segmentation Based on Ensemble Learning and Improve GrowCut Techniques
}

Fatemeh Jafari

islamic azad university of ferdows

Hamidreza Ghaffari ( $\nabla$ hghaffari_papers@ferdowsiau.ac.ir )

islamic azad university of ferdows https://orcid.org/0000-0002-7437-8466

\section{Research}

Keywords: MRI Brain, GrowCut, Ensemble learning, Image processing

Posted Date: February 15th, 2021

DOI: https://doi.org/10.21203/rs.3.rs-194240/v1

License: (c) (1) This work is licensed under a Creative Commons Attribution 4.0 International License.

Read Full License 


\title{
EL-IGrowCut: MRI Brain Tissue Segmentation Based on Ensemble Learning and Improve GrowCut Techniques
}

\author{
Fatemeh Jafari, Hamidreza Ghaffari * \\ Department of Computer Engineering, Ferdows Branch, Islamic Azad University, Ferdows, Iran . \\ *Corresponding Author Email: hghaffari_papers@ferdowsiau.ac.ir
}

\begin{tabular}{|c|c|}
\hline PAI & ABSTRACT \\
\hline $\begin{array}{l}\text { Article history: } \\
\text { Received } \\
\text { Received in revised } \\
\text { form } \\
\text { Accepted } \\
\text { Available online }\end{array}$ & 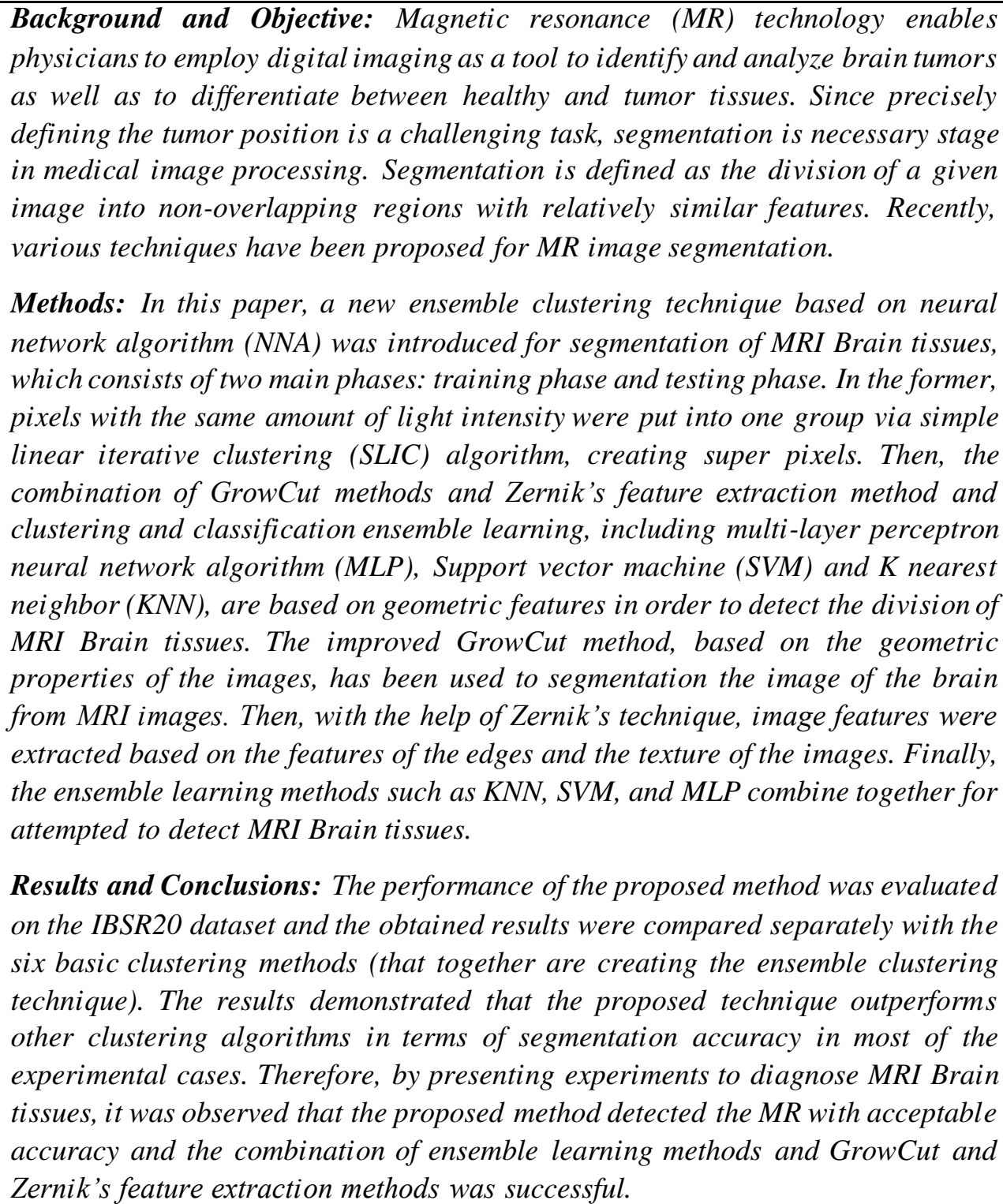 \\
\hline
\end{tabular}




\section{INTRODUCTION}

Medical imaging is one of the useful tools widely used to locate and analyze brain tumor tissues as well as differentiate between healthy and tumor tissues [1]. Magnetic resonance imaging (MRI) technology is a type of medical imaging that is used in clinical diagnosis and is of better quality compared to the computed tomography (CT) [2]. MRI enables physicians to get high quality, high resolution digital imaging via the combination of magnetic fields and radio waves. Important information about the brain tissues found in these images allows for more detailed investigations within the brain pathology. Determining the exact location of a tumor is a challenging and effective task for treatment. Thus, segmentation of an image is an important step in medical imaging processing. Image segmentation is typically defined as division of the image into non-overlapping regions with relatively similar features [3]. In the segmentation of brain images, the brain image is divided into three main tissues: white matter (WM), gray matter (GM) and cerebrospinal fluid (CSF) [4]. Fig. 1 and Fig. 2 depict a typical and segmented MRI Brain image, respectively. The purpose of segmentation is to simplify the original image and convert it to another format to be easily analyzed and perceived. Brain image segmentation is used to determine the boundaries between brain tissues as well as label assignment to each pixel in the image.

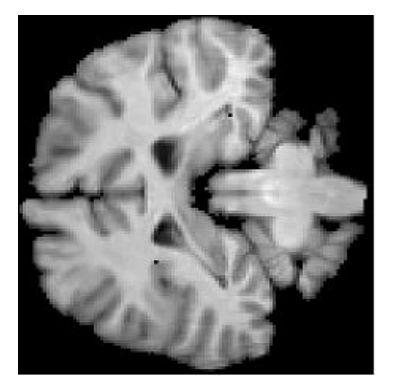

Figure 1. Original MR Image

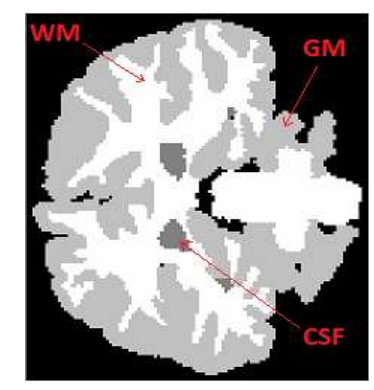

Figure 2. Segmented Image

In recent years, several methods have been proposed for image segmentation based on clustering techniques [5-7]. Clustering is used as one of the most common and effective techniques for image segmentation. The k-means algorithm [8], fuzzy c-means (FCM) [2, 9] and self-organizing mapping (SOM) algorithm have been popular clustering techniques with their own advantages and disadvantages [2, 9]. Researchers have also reported the application of meta-heuristic algorithms such as genetic algorithm (GA) $[10,11]$, artificial bee colony (ABC) [12-14], ant colony optimization (ACO) $[15,16]$, particle swarm optimization (PSO) [17] and differential evolution algorithm (DE) [18] to cluster and/or segment medical images [19].

In this paper, an ensemble clustering method based on NNA for MRI Brain tissue segmentation was proposed that consists of two main training and testing phases. In the first phase, a certain number of datasets are fed into model as training data. Next, the pixels with the similar light intensity are grouped by 
an algorithm, leading to the formation of super pixels. Afterwards, we used an improved GrowCut fuzzy algorithm at this stage to plot the MRI in the main image, this algorithm is also used by the GrowCut fuzzy fragmentation method. So, Zernik's feature extraction is performed and feature vector is created, these super pixels are divided into three clusters and labeled by the ensemble clustering technique. Using this feature matrix, ensemble learning model is trained, which is used in the testing phase to segment test images. In the testing phase, the feature labels extracted for the test image are predicted by the trained ensemble learning model, resulting in the increased speed of execution and segmentation by eliminating the clustering step. The performance of the proposed method was evaluated on the IBSR20 dataset and the obtained results were separately compared with six clustering algorithms (i.e., neural network algorithm (NNA), particle swarm optimization (PSO), artificial bee colony (ABC) and genetic algorithm (GA), K-means clustering, and self-organizing mappings (SOMs)) and other classification algorithms. The remainder of this paper is categorized as follows: Section 2 deals with past work, is described in Section 3 of the suggested model and proposed architecture. In Sections 4 and 5, the results are presented and the final conclusions are presented.

\section{RELATED WORKS}

So far, several methods have been reported for segmentation of MR images. One of these methods operating automatically is the brain image clustering method using k-means technique which detects the shape and spectrum of tumors. A median filter is used to eliminate artifacts and sharpen the edges of the image. Then, the k-means clustering method is applied using centroid random values. A binary mask is used to predict and calculate the tumor region in the image in order to identify a class with higher contrast values [8]. Fuzzy clustering techniques are also used to segment MR images [2, 9]. In a segmentation method based on theoretical learning of information is proposed for supervoxel level segmentation. Information theoretic discriminative segmentation (ITDS) is a clustering method for analyzing contrast data and selecting brain tissue features at the level of super pixel, both requiring duplicate adjustments to produce more classification accuracy [20]. The routine of these adjustments requires a great deal of time to produce a good classification. In the concept of fuzzy learning and deep learning were introduced by providing a fuzzy deep neural network (FDNN) to extract information from fuzzy and neural representation simultaneously [21]. The combination of knowledge gained from deep learning and fuzzy logic was used for final illustration of the intended data for classification. The merits of this method is to reduce the uncertainties and eliminate the noise in the original data. In two unsupervised methods were designed to segment MRI Brain images using SOM [22]. The first approach is called self-organizing mapping (SOM) with histogram based fast segmentation (HFS-SOM). In HFS-SOM, the features are extracted from the image histogram. Feature vectors consist of intensity generation probability, relative position with respect to intensity, mean probability values in a 3-bin window and the variance of that window used for SOM training. Then, k-means is used to cluster the SOM output layer. The second approach is known as SOM with entropy gradient segmentation (EGS-SOM). The first- and second-order statistical features are extracted from overlapping windows of $7 * 7$ pixels. For SOM training, a genetic algorithm (GA) is applied to select the best differentiating features. Finally, SOM outputs are clustered using the efficient EGS algorithm. The HFS-SOM method is faster because it does not need to adjust any parameters; while, the latter is more stable under noisy and improper intensities. In a hybrid MR segmentation method was proposed that uses discrete wavelet transform (DWT) and FCM to eliminate heterogeneities [23]. DWT is applied to the MR input image to obtain the LL, LH, HL and HH subgroups. To obtain the sharpened image, the estimation coefficients of the LL subgroup are set to zero. Then, the inverse discrete wavelet conversion is applied to obtain a robust image. The resulting image is segmented using the FCM technique. Finally, the Kirch edge detection mask is used to fill in missed edge information and improve the output image. In an automatic segmentation method of brain MR images was presented. For each label, the voxel intensities 
of all MRI sequences are modeled using the Gaussian distribution [24]. The Gaussian distribution parameters are evaluated as the maximum likelihood probability and the final probability of each label is determined using Bayesian estimation. Regional intensity, tissue, voxels' location, and estimated last probability are used to classify each voxel into one of four classes (CSF, GM, WM, and background) along with a multi-vector support machine. In this paper, an ensemble clustering technique is introduced to take the advantages of some existing clustering techniques in order to obtain accurate segmentation of brain tissues. This method is inspired by the method presented in that is tried to improve its performance with some modifications. The next section examines the proposed method [25].

\section{The proposed method}

In Figure 1, the flowchart shows the steps in the proposed method. In this paper, we will first try to perform all the MRI Brain Tissue Segmentation by the proposed system include improve GrowCut segmentation algorithm, Zernik feature extraction, ensemble learning and do not need to diagnose the physician. To do this, we first used an improved GrowCut fuzzy algorithm at this step to plot the median MRI Brain scan area in the main image, this algorithm is also used by the GrowCut fuzzy fragmentation method. In the next step, we use the Zernik feature extraction algorithm to obtain the characteristics of the segmental fraction in the previous step, as well as in the last step using the combination of the method Classification of SVM and MLP and KNN (Ensemble learning system) in the classification step as well as the final vote of the output What can be detected with high accuracy and MRI Brain Tissue Segmentation images achieved. Finally, we propose a powerful and effective model for dividing MRI Brain images. 


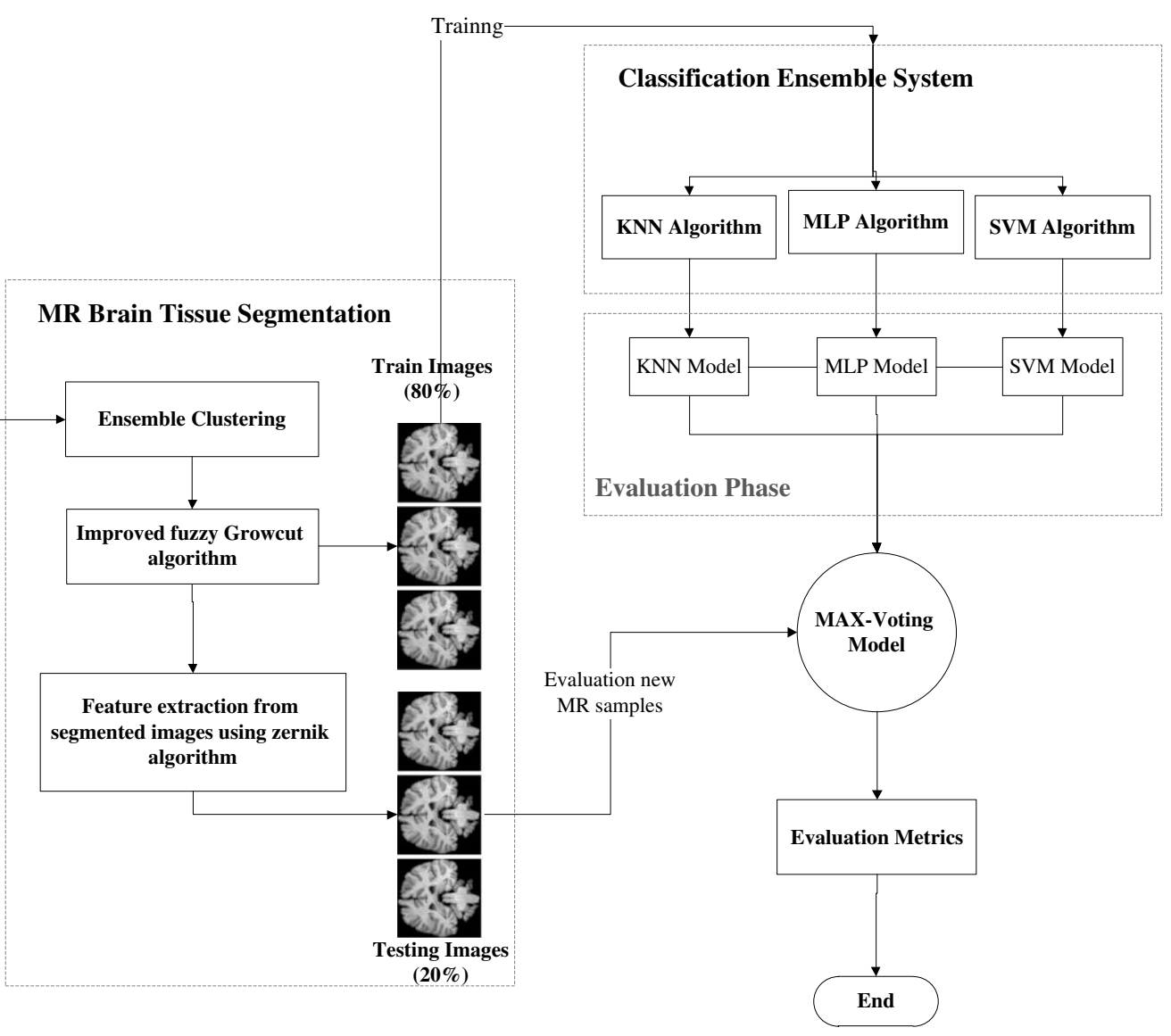

Figure 1. The proposed Architecture

The most important parts of the proposed method in this research will be presented below the relevant sections. The proposed method includes the sections discussed below.

\subsection{Ensemble clustering}

A neural network algorithm (NNA)-based clustering technique along with five other clustering techniques called k-means, FCM, SOM, GA and PSO are employed for clustering the features obtained during the feature extraction phase. And, the input image is segmented into three clusters using each of these methods by considering the segmented image available in the dataset. Then, using the post processing method presented in [25], the clustering result is improved. In [25], post processing is done in the last test step. However, because the feature vector created in this phase is fed into SVM as a training dataset, data with the wrong labels will eventually lead to the advent of an inappropriate classification. So, post processing is also employed in this phase. Finally, the input image is segmented into three clusters by a single ensemble clustering algorithm. In this paper, the ensemble clustering technique presented in [27] is employed.

\section{Initial population generation}


In the NNA algorithm, like the other meta-heuristic algorithms such as GA, the solutions are presented in vector form that are the components representing the dimensions of the solution space. In the GA, these solutions are called chromosomes, which can be represented by

$$
\text { pattern solution }=\left[\mathrm{x}_{1} \cdot \mathrm{x}_{2} \ldots \ldots \mathrm{x}_{\mathrm{D}}\right]
$$

The first step in this algorithm is to generate the initial population, comprising of $\mathrm{N}_{\text {pop }}$ solution vectors, randomly generated in the solution space. So, the initial population is a two-dimensional matrix $\left(\mathrm{N}_{\text {pop }} \times \mathrm{D}\right)$ in which its rows represent the number of solutions and its columns denote the problem space dimension.

$$
\mathrm{X}=\left[\begin{array}{ccccc}
\mathrm{x}_{1}^{1} & \cdots & \mathrm{x}_{\mathrm{i}}^{1} & \cdots & \mathrm{x}_{\mathrm{D}}^{1} \\
\mathrm{x}_{1}^{2} & \cdots & \mathrm{x}_{\mathrm{i}}^{2} & \cdots & \mathrm{x}_{\mathrm{D}}^{2} \\
\vdots & \cdots & \vdots & \cdots & \vdots \\
\mathrm{x}_{1}^{N_{p o p}} & \cdots & \mathrm{x}_{\mathrm{i}}^{N_{p o p}} & \cdots & \mathrm{x}_{\mathrm{D}}^{N_{p o p}}
\end{array}\right]
$$

where the values of each component in the solutions can be continuous or binary. The fitness or cost of each solution is calculated by the objective function $\mathrm{f}$.

$$
C_{i}=f\left(x_{1} \cdot x_{2} \ldots . x_{D}\right) \quad i=1.2 \ldots . N_{\text {pop }}
$$

Where $C_{\mathrm{i}}$ represents the value of the $i$ th solution's objective function in the population. After calculating the value of the cost function for all available solutions in the population, the best solution is considered as target solution ( $\left.\mathrm{X}^{\text {target}}\right)$. The best solution in the minimization problem is the one with the least cost function. Now, once the best solution is determined as mentioned above, it is required to push the rest of the solutions toward the best solution. For this purpose, a weighting matrix (W) is expressed in the next step via the mathematical concepts and relationships found in ANNs. Using this matrix, the population matrix can be updated for subsequent iterations so that the solutions converge to the best solution.

\section{Weighting matrix $(W)$}

The initial weighting matrix (W) is made up of random numbers in the interval [0 and 1] that are updated in each subsequent iteration of the algorithm in the same interval. The weighting matrix $\mathrm{W}$ in the th iteration can be represented by (4).

$$
\mathrm{W}(\mathrm{t})=\left[\mathrm{W}_{1} \cdot \mathrm{W}_{2} \ldots \cdot \mathrm{W}_{\mathrm{N}_{\mathrm{pop}}}\right]
$$




$$
\begin{aligned}
& =\begin{array}{ccccc}
\mathrm{w}_{1}^{1} & \cdots & \mathrm{w}_{1}^{\mathrm{i}} & \cdots & \mathrm{w}_{1}^{\mathrm{N}_{\text {pop }}} \\
\mathrm{w}_{2}^{1} & \cdots & \mathrm{w}_{2}^{\mathrm{i}} & \cdots & \mathrm{w}_{2}^{\mathrm{N}_{\text {pop }}} \\
\vdots & \cdots & \vdots & \cdots & \vdots \\
\mathrm{w}_{\mathrm{N}_{\mathrm{pop}}}^{1} & \cdots & \mathrm{w}_{\mathrm{N}_{\mathrm{pop}}}^{\mathrm{i}} & \cdots & \left.\mathrm{w}_{\mathrm{N}_{\mathrm{pop}}}^{\mathrm{N}_{\text {pop }}}\right\rfloor
\end{array} \\
& \begin{array}{llllll}
\mathrm{w}_{11} & \cdots & \mathrm{w}_{\mathrm{i} 1} & \cdots & \mathrm{w}_{\mathrm{N}_{\mathrm{pop}} 1}
\end{array} \\
& =\begin{array}{ccccc}
\mathrm{w}_{11} & \cdots & \mathrm{w}_{\mathrm{i} 2} & \cdots & \mathrm{w}_{\mathrm{N}_{\mathrm{pop}} 2} \\
\vdots & \cdots & \vdots & \cdots & \vdots \\
\mathrm{w}_{11} & \cdots & \mathrm{w}_{\mathrm{iN}} & \cdots & \left.\mathrm{w}_{\mathrm{N}_{\mathrm{pop}} \mathrm{N}_{\mathrm{pop}}}\right\rfloor
\end{array}
\end{aligned}
$$

Matrix $\mathrm{W}$ is a square matrix with a dimension of $\mathrm{N}_{\mathrm{pop}} * \mathrm{~N}_{\mathrm{pop}}$ that is randomly generated in uniform shape in the abovementioned interval with the limitations expressed in (4).

$$
\begin{aligned}
& \sum_{j=1}^{N_{\text {pop }}} w_{\text {ij }}(t)=1 . \quad i=1.2 .3 . \ldots . N_{\text {pop }} \\
& w_{\text {ij }} \in U(0.1) \quad i . j=1.2 .3 \ldots . N_{\text {pop }}
\end{aligned}
$$

The purpose of considering these two constraints for weighting values is to control the difference between the new solutions produced among the existing solutions. In fact, the aforementioned constraint leads the generated solutions with long steps to not detract from the existing solutions. And, its effect is like that of pheromone values in the ant colony algorithm. Once weighting matrix is constructed, new solutions are generated using (5) to update the population matrix.

$$
\begin{aligned}
& X_{j}^{\text {new }}(t+1)=\sum_{i=1}^{N_{\text {pop }}} w_{i j}(t) \times X_{i}(t) . \quad j=1.2 .3 \ldots . N_{p o p} \\
& X_{i}(t+1)=X_{i}(t)+X_{j}^{\text {new }}(t+1) . \quad i=1.2 .3 \ldots N_{p o p}
\end{aligned}
$$

where $t$ represents the iteration number of the algorithm. After the new solutions are generated using the solutions obtained in the previous step, the weighting matrix $\mathrm{W}$ based on the best weight ( $\mathrm{W}^{\text {target}}$ ) corresponding to the best solution is updated by (6).

$$
\mathrm{W}_{\mathrm{i}}^{\text {updated }}(\mathrm{t}+1)=\mathrm{W}_{\mathrm{i}}(\mathrm{t})+2 \times \operatorname{rand} \times\left(\mathrm{W}^{\text {target }}(\mathrm{t})-\mathrm{W}_{\mathrm{i}}(\mathrm{t})\right)
$$

After updating the weighting matrix, the constraints (1) should be applied.

\subsection{MRI fragmentation with improved Grow Cut algorithm}

One of the most important applications of this algorithm that is used to improve the results of this paper is that, when processing MRI BRAIN images, it can segment the MRI BRAIN range to be identified at a later stage with higher sensitivity. In this section, we first discuss the theoretical analysis of the GrowCut fuzzy algorithm and then describe the segmentation of the MRI BRAIN images precisely.

A dual-axial deterministic cellular machine is a triangular $A=(S . N . \delta), S$ is a non-zero state set, and $N$ is a neighboring system, and $\delta: S^{\wedge} N \rightarrow S$ is a local transfer function. This function is the rule of calculating 
cell states in time $t+1$ and it captures the states of adjacent cells in the past t [16]. A digital image is a twodimensional array of $K \times m$ pixels. A non-sticking image can be considered as a specific configuration of the cellular machine, where the cell space $P$ is represented using a set of arrays with $\mathrm{K} \times \mathrm{m}$ and the initial states of $\forall p \in P$ that shown in Eq. (8).

$$
l_{p}=R G B_{p}
$$

Where $R G B_{p}$ is a three-dimensional p-pixel color vector in RGB space. The ultimate goal of segmentation is to assign each pixel to one of the $K$ labels. When the user starts the segmentation process according to the pixels in the image, the labels of the desired cells will be given. This process is the primary mode of the cellular machine [37]. Att +1 , the label of the cell $l_{p}{ }^{(t+1)}$ with the force $\theta_{p}{ }^{(\mathrm{t}+1)}$ will be determined using the Eq. (9):

$$
\mathrm{g}(\mathrm{x})=1-\frac{\mathrm{x}}{\max \|\overrightarrow{\mathrm{C}}\| 2}
$$

Where $g$ is a uniform reduction function in the interval $[0,1]$, which will be calculated by Eq. (9). The pixel tagging process can be likened to the growth and overcome of the bacterial $K$ type. Bacteria start to grow from target pixels and try to occupy the entire image. That's why it's called GrowCut. The rules for growing competition between bacteria are clear. At each separate step, each cell tries to attack its adjacent cells. The attack force is determined by the attacker's force of the $\theta_{q}$ cell and the distance between the attacker and the defender is determined by the vectors $C, \theta$ and $C \cap p$. If the attacker's force is greater than the defender's force, the defending cell will fail, and the label and force will change. The result of this local competition is that it is the strongest bacteria occupies the cells around it and will be successfully dispersed on the image surface [37].

\subsection{Feature extraction using Zernik}

In this paper, for the purpose of extracting the properties of MRI BRAIN images, the Zernik wax method has been used because of its robustness to image rotation and simplicity of calculation [38] [39]. This method is one of the most important feature extraction methods based on geometric patching. This research has been considered. The German Zernik polynomial is defined as Eq. (10) [39].

$$
\mathrm{V}_{\mathrm{nm}}(\mathrm{x}, \mathrm{y})=\mathrm{V}_{\mathrm{nm}}(\rho \cdot \theta)=\mathrm{R}_{\mathrm{nm}}(\rho) e^{j m \theta}
$$

In which $m$ and $n$ are the polynomials of the vertex Zernik. $N$ is a positive integer or zero, and $m$ is a positive integer or negative, so that $n m$ the number is paired and $m n \geq 0$. $\rho$ is the vector length between the center of the circle and the pixel $(\mathrm{x}, \mathrm{y})$ of the image. $\theta$ is the angle between the vector length $\rho$ and the $x$ axis in the counterclockwise direction. $R_{m n}(\rho)$ is a vertical radial polynomial with the real value calculated by Eq. (11).

$$
\mathrm{R}_{\mathrm{nm}}(\rho)=\sum_{s=0}^{(n-|m|) / 2} \frac{(-1)^{s}[(n-s) !] \rho^{n-2 s}}{s !\left(\frac{n+|m|}{2}-s\right) !\left(\frac{n-|m|}{2}-s\right) !}
$$


The resonant matrix refers to the projection of the image $\mathrm{f}(\mathrm{x}, \mathrm{y})$ in the image on the polynomial $V_{n m}(\mathrm{x}$, $y$ ), and the nth order is defined by $m$ repetition of the Zernik waistband by Eq. (12):

$$
\mathrm{Z}_{\mathrm{nm}}=\frac{n+1}{\pi} \iint_{x^{2}+y^{2} \leq 1}^{1} f(x, y) V n m^{*}(x \cdot y) d x d y
$$

The Zernik is defined in polar coordinates by Eq. (13):

$$
\mathrm{Z}_{\mathrm{nm}}=\frac{n+1}{\pi} \int_{0}^{2 \pi} \int_{0}^{1} f(\rho . \theta) \rho d \rho d \theta
$$

Domain $Z_{n m}$ It is considered as a fixed feature against the rotation of the image. To get the domain and phase, first the image is converted into gray space, then the image completes the gray image, and the resulting complement is converted to binary space, and at the end, the Zernik mist will be calculated from the binary image. According to the above description, the Zernik feature extraction algorithm performs steps to extract features from segmented images. These steps in the proposed method to extract the features of the images are:

1. All segmented images by the GrowCut fuzzy segmentation algorithm are entered as inputs to the feature extraction system.

2. Characteristic extraction algorithm receives one-to-one images individually and performs a feature extraction process on them. With each image received, based on the kernel and the relationships defined for this algorithm, features of each image are extracted numerically.

3. Numerical features in the form of vectors and matrices are presented as outputs by Zernik's algorithm.

4. Steps 2 and 3 continue until all input images are analyzed and their features extracted.

Therefore, the output of this part is a set of digits and attributes that are trained and tested by fragmentation algorithms, and ultimately the presence of MRI Brain images is detected. In the next section, after separating the educational and Testing images, we will focus on how to combine the SVM, KNN, and MLP classifications algorithms in ensemble system.

\subsection{Separation of Training and Testing images}

After fragmentation of the MRI Brain, all data should be divided into two parts. Training data, which is $80 \%$ of the total MRI Brain images, and Testing data, which is $20 \%$ of the MRI Brain datasets. Training data is used to train and generate models using ensemble learning system such as KNN, SVM, and MLP. testing data is used to evaluate and measure the performance of the proposed method.

\subsection{Ensemble learning system}

In this section, we present the logic of the proposed method in the last step to classify the diagnosis and division of MRI Brain images. The final output of the proposed system is that the image inserted into the proposed system has a MRI Brain images. If the MRI BRAIN images does not correct, the error metric is 
plus score, and if it has a correct MRI Brain images, then it is examined whether MRI Brain images are benign or malignant. In Figure 2, a general overview of how the ensemble learning system responses are used in this research is shown.

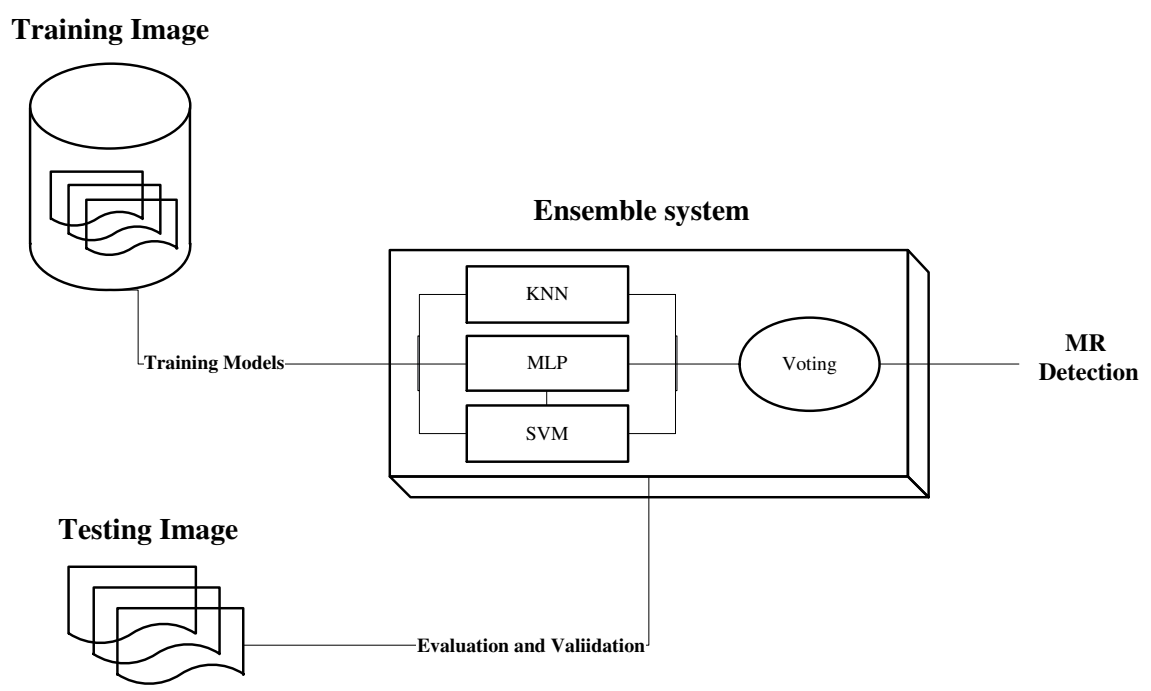

Figure 2. Flowchart of ensemble system for MRI Brain images.

As can be seen from the Fig. 2, the training data is firstly added to the single machine learning algorithms in ensemble learning system. Each algorithm generates a model in isolation in accordance with its logic. Then, to each model, test samples are introduced to evaluate and measure the accuracy of the proposed method. Then each algorithm issues an answer for each sample. Existing responses indicate that the imported specimen has a MRI Brain images. Finally, all responses will be entered into a Voting system. The Voting system finds the maximum number of candidates and sends out the final response. Given that our main goal in this study is to increase the accuracy of the diagnosis and division of MRI Brain images, the Max core is used in the Voting process. In general, we can combine the classification algorithms in the proposed method in the form of steps. These steps are generally presented below:

1) Training images are firstly introduced into the MLP classification algorithm. This algorithm performs model training based on the imported samples and generates a model. The generated model has rules and structures based on sample images.

2) Training images are first entered into the SVM classification algorithm. This algorithm performs model training based on the imported samples and generates a model. The generated model has rules and structures based on sample images.

3) Training images are first entered into the KNN Classification Algorithm. This algorithm performs model training based on the imported samples and generates a model. The generated model has rules and structures based on sample images. 
4) After the steps 1,2 and 3 are fully applied to train images, test images are entered into the input of each model. At this step, the models are completely structured and can apply the diagnostic process to test images. Therefore, each model executes the detection process on any sample of Testing MRI Brain images. The output of each model is a response. The corresponding answer is whether the image has a MRI Brain images of the brain. If the MRI Brain images is benign or malignant.

5) Step 4 runs for all SVM, KNN, and MLP algorithms. So, so far, there are three different or identical answers from three separate algorithms. These replies are entered into the Voting system in the form of three numbers. The Voting system uses a Max strategy to respond to a given poll. The answer that has the most candidate is considered as the main answer to the proposed system.

6) Steps 1 through 5 continue until all Testing images have been analyzed.

\section{Results and Discussion}

In this section, the proposed method is compared with other methods so that, first, the test settings are laid out, then the benchmarks are set out and finally, the experiments are analysed.

\subsection{Experimental Setup}

The proposed method is implemented using the MATLAB simulator version 2015a. The operating system is Windows 10, the operating system is of the 32-bit type, 4GB of RAM is used from which 3.06GB is usable, with 7-core Intel processor (Core TM) i7 CPU) - Q 720 and processor base frequency of @ $1.60 \mathrm{GHz}$.

\subsection{Dataset}

In this study, an image dataset from the Internet Brain Segmentation Database (IBSR) is considered that is prepared at the Massachusetts Hospital Morphometric Analysis Center (http://www.cma.mgh.harvard.edu/ibsr/). The MRI image database consists of two datasets for dividing brain tissue into normal samples, one with 20 samples and the other with 18 samples. The first and second datasets are known as IBSR20 and IBSR18, respectively. Here, IBSR20 is used in this study. In this dataset, the images are numbered sequently. This twenty images have been divided into two training and testing phases. First five images are considered as training data and the rest 15 images are taken as testing data. The reason why the images are divided in this way is to get a fair comparison between our method and the segmentation algorithm reported in [25]. The IBSR20 dataset contains an MRI Brain images' dataset with the weight of T1 for 20 normal samples and their associated manual segmentation. The size of voxel network is $256 * 256 * 65$ with a thickness of $3.1 \mathrm{~mm}$. The precise manual segmentation of WM, GM and CSF available in the dataset are used to evaluate the obtained results. The proposed model was applied for segmentation on training and testing images where the obtained results illustrated further in diagrams and tables. In addition, all the steps of the proposed model are fully applied to one of the dataset images, and the output of each step is shown graphically. Fig. 3 depicts the testing image in the dataset, Fig 4 illustrates the correct segmentation image for test image, and Fig. 5 shows the output of the SLIC algorithm. Fig. 6-10 show the results for the clustering algorithms. And finally, Error! Reference source not found. illustrates the result of the ensemble clustering algorithm. Obviously, the segmentation obtained by the proposed algorithm and NNA algorithm is closely similar to ground truth compared to the other algorithms.

\subsection{Performance Metrics}


To evaluate the accuracy of the segmentation methods, two criteria, i.e. the Jacquard similarity (JS) and Dice Similarity Coefficient (DSC), were used. JS is calculated as the ratio between the intersection and union of the images segmented by the algorithm and the ground truth algorithm. The JS spectrum varies in between zero and unity. Higher JS values indicate that the segmented region is more in line with the criterion region. JS is determined by Eq. (7)[25].

$$
\mathrm{JS}=\frac{\mathrm{S}_{1} \cap \mathrm{S}_{2}}{\mathrm{~S}_{1} \cup \mathrm{S}_{2}}
$$

where $S_{1}$ is the benchmark image and $S_{2}$ is the segmented image. Another criterion named DSC was also employed in order to evaluate the performance of the proposed algorithm. DSC is a statistical validation criterion employed for assessing the accuracy of the segmentation methods in[25]. The overlap between the image of ground truth and the segmented image by the algorithm is expressed by (8):

$$
\text { DSC }=2 \times \frac{S_{1} \cap S_{2}}{\left|S_{1}\right| \cup\left|S_{2}\right|}
$$

where |. | denotes cardinal.

Also, the criteria metrics used for classification in this paper to compare the proposed method with other methods are:

$$
\text { Precision }=\frac{\mathrm{TP}}{T P+\mathrm{FP}}
$$

Eq. (16) is used to check the accuracy of the classification ensemble learning proposed method.

$$
\text { ReCall }=\frac{\mathrm{TP}}{\mathrm{TP}+\mathrm{FN}}
$$

Eq. (17) indicates the recall of the proposed method where the False Negative (FN) parameter indicates the number of samples diagnosed as normal.

$$
\text { Accuracy }=\frac{\mathrm{TP}+\mathrm{TN}}{\mathrm{TP}+\mathrm{TN}+\mathrm{FP}+\mathrm{FN}}
$$

Eq. (18) is used to calculate the accuracy where the only parameter not described is True Negative (TN) representing the number of samples correctly identified as normal. Finally, the error rate of the proposed method is calculated based on the Eq. (18).

In these equations, $\mathrm{TP}$ is the number of positive integers, $\mathrm{TN}$ is the negative integer, $\mathrm{FT}$ is the number of positive errors and FN is the number of negative errors in MS detection in sample MRI images.

Table. 3 provides the JS results using k-Means, FCM, SOM, EBTs and GA, PSO, NNA and, finally, ensemble clustering on training dataset. EBTs (efficient fully-automatic brain tissue segmentation) algorithm is referred to the method reported in [25]. As mentioned earlier, first 5 images in database are considered as the training images and the rest are testing images. The results by the algorithms are obtained in terms of each five training images (due to space limitation, the table is divided into A and B parts). It is observed that the proposed method has higher similarity for all three classes (WM, GM and CSF) in most cases compared to the other six classification methods. On the other hand, it was found that other methods worked well for the WM and GM classes but were less effective for the CSF class.

TABLE 3 (A). The JS results obtained by application of the algorithms on training dataset 


\begin{tabular}{ccccccccccccc}
\hline \multicolumn{3}{c}{ K-means } & \multicolumn{3}{c}{ FCM } & \multicolumn{3}{c}{ SOM } & \multicolumn{3}{c}{ EBTs } \\
\hline$C S F$ & $G M$ & $W M$ & $C S F$ & $G M$ & $W M$ & $C S F$ & $G M$ & $W M$ & $C S F$ & $G M$ & $W M$ \\
0.1820 & 0.6567 & 0.6906 & 0.1775 & 0.6749 & 0.6997 & 0.1692 & 0.6779 & 0.7018 & 0.2801 & 0.7319 & 0.7300 \\
0.2453 & 0.5051 & 0.4726 & 0.2430 & 0.5062 & 0.4914 & 0.2416 & 0.5036 & 0.4706 & 0.2445 & 0.5161 & 0.4741 \\
0.6264 & 0.6472 & 0.6367 & 0.5652 & 0.6656 & 0.6459 & 0.6264 & 0.6476 & 0.6373 & 0.6924 & 0.6947 & 0.6623 \\
0.4489 & 0.6299 & 0.5742 & 0.4674 & 0.6189 & 0.5700 & 0.4489 & 0.6277 & 0.5718 & 0.5040 & 0.6408 & 0.5792 \\
0.4665 & 0.7392 & 0.6556 & 0.3672 & 0.6676 & 0.6527 & 0.4439 & 0.7379 & 0.6556 & 0.4993 & 0.7648 & 0.7084 \\
\hline
\end{tabular}

TABLE $3(\mathbf{B})$. The JS results obtained by application of the algorithms on training dataset

\begin{tabular}{cccccccccccc}
\hline & GA & \multicolumn{3}{c}{ PSO } & \multicolumn{3}{c}{} & \multicolumn{3}{c}{ NNA } & \multicolumn{4}{c}{ Ensemble Clustering } \\
\hline$C S F$ & $G M$ & $W M$ & $C S F$ & $G M$ & $W M$ & $C S F$ & $G M$ & $W M$ & $C S F$ & $G M$ & $W M$ \\
0.2724 & 0.7142 & 0.7187 & 0.3236 & 0.7634 & 0.7360 & 0.3201 & 0.7452 & 0.7251 & 0.5404 & 0.8259 & 0.8220 \\
0.2409 & 0.5021 & 0.4651 & 0.2123 & 0.5156 & 0.4784 & 0.3927 & 0.5282 & 0.4621 & 0.4601 & 0.7453 & 0.7158 \\
0.6864 & 0.6429 & 0.6362 & 0.6864 & 0.7017 & 0.6602 & 0.6129 & 0.6824 & 0.6508 & 0.7570 & 0.8188 & 0.7835 \\
0.4754 & 0.6200 & 0.5729 & 0.4524 & 0.6430 & 0.5785 & 0.3909 & 0.6378 & 0.5854 & 0.5956 & 0.7928 & 0.7499 \\
0.4658 & 0.7252 & 0.6865 & 0.4260 & 0.7396 & 0.7026 & 0.4202 & 0.7029 & 0.6784 & 0.5082 & 0.7973 & 0.7720
\end{tabular}

Tables 4-7 provide the average results for evaluation criteria of JS and DSC by application of the aforementioned segmentation algorithms on training and testing dataset for WM, GM, and CSF classes. The spatial overlap metric has no impact on estimation of the overall performance in terms of error. Thus, the obtained values of sensitivity, specificity and accuracy are presented for each method in Table 8. The quantitative results demonstrated that the proposed method outperforms the other base clustering techniques for WM, GM and CSF tissues.

TABLE 4. JS values for segmentation algorithms for training and testing IBSR20 dataset

\begin{tabular}{ccccc}
\hline class & k-means & FCM & SOM & EBTs \\
\hline CSF & 0.3127 & 0.2944 & 0.3118 & 0.3884 \\
GM & 0.7000 & 0.6760 & 0.6867 & 0.7253 \\
WM & 0.6593 & 0.6567 & 0.6525 & 0.6895 \\
Average & 0.5573 & 0.5424 & 0.5503 & 0.6011 \\
\hline
\end{tabular}

TABLE 5. JS values for segmentation algorithms for training and testing dataset 


\begin{tabular}{cccccc}
\hline Dataset & class & GA & PSO & NNA & Ensemble Clustering \\
\hline \multirow{4}{*}{ IBSR20 } & CSF & 0.3884 & 0.3791 & 0.3789 & 0.5145 \\
& GM & 0.7041 & 0.7003 & 0.7082 & 0.8103 \\
& WM & 0.6660 & 0.6727 & 0.6698 & 0.7978 \\
& Average & 0.5847 & 0.5841 & 0.5857 & 0.7075 \\
\hline
\end{tabular}

TABLE 6. DSC values for segmentation algorithms for training and testing dataset

\begin{tabular}{llllll}
\hline Dataset & class & k-means & FCM & SOM & EBTs \\
\hline \multirow{4}{*}{ IBSR20 } & CSF & 0.4631 & 0.4427 & 0.4626 & 0.5459 \\
& GM & 0.8212 & 0.8055 & 0.8123 & 0.8385 \\
& WM & 0.7930 & 0.7911 & 0.7880 & 0.8140 \\
& Average & 0.6924 & 0.6798 & 0.6876 & 0.7328 \\
\hline
\end{tabular}

TABLE 7. DSC values for segmentation algorithms for training and testing dataset

\begin{tabular}{cccccc}
\hline Dataset & class & GA & PSO & NNA & Ensemble Clustering \\
\hline \multirow{4}{*}{ IBSR20 } & CSF & 0.5407 & 0.5363 & 0.5373 & 0.6729 \\
& GM & 0.8239 & 0.8219 & 0.8269 & 0.8949 \\
& WM & 0.7973 & 0.8021 & 0.8001 & 0.8870 \\
& Average & 0.7206 & 0.7201 & 0.7215 & 0.8183 \\
\hline
\end{tabular}

TABLE 8. The average segmentation accuracy of algorithms on training and testing dataset

\begin{tabular}{cccc}
\hline Algorithms & Sensitivity & Specificity & Accuracy \\
\hline k-means & 0.9340 & 0.9602 & 0.8354 \\
FCM & 0.9264 & 0.9550 & 0.8185 \\
SOM & 0.9230 & 0.9526 & 0.8113 \\
EBTs & 0.9209 & 0.9506 & 0.8092 \\
GA & 0.9220 & 0.9517 & 0.8100 \\
PSO & 0.9213 & 0.9511 & 0.8090 \\
NNA & 0.9205 & 0.9504 & 0.8080
\end{tabular}




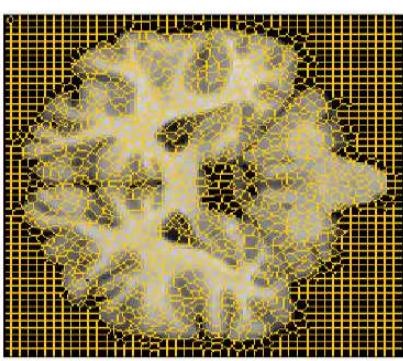

Figure 3. Test image

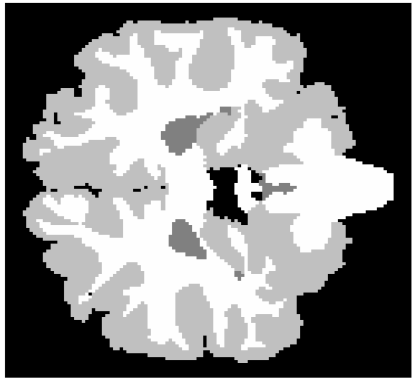

Figure 4. Ground truth

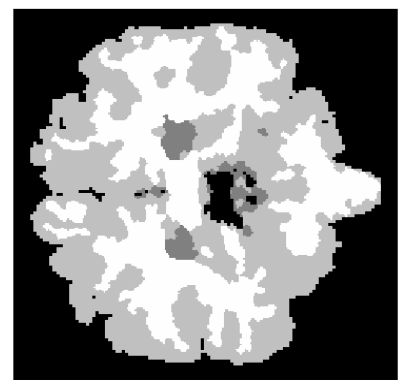

Figure 9. GA

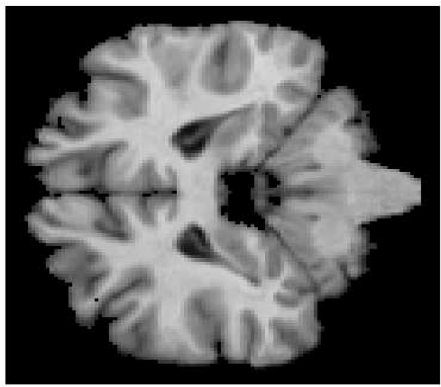

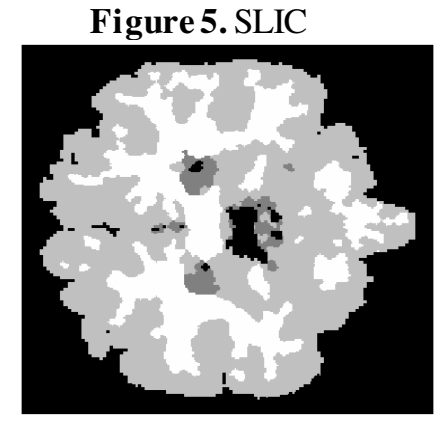

Figure 6. K-means

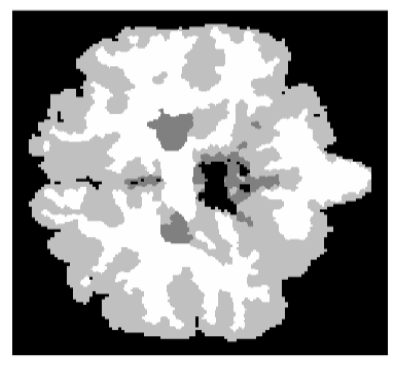

Figure 13. NNA
0.9519

0.9726

0.8737

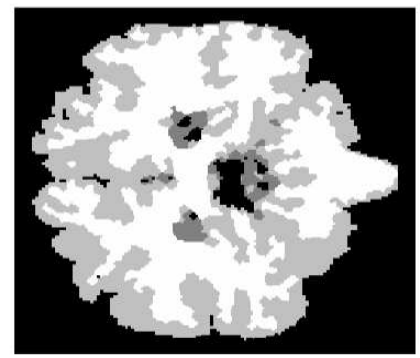

Figure 7. FCM

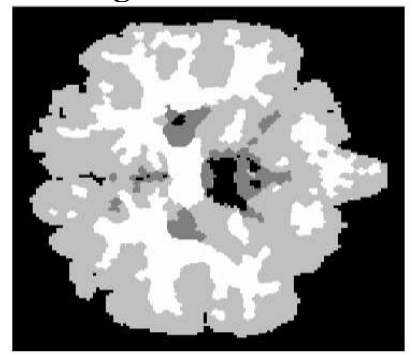

Figure 8. SOM

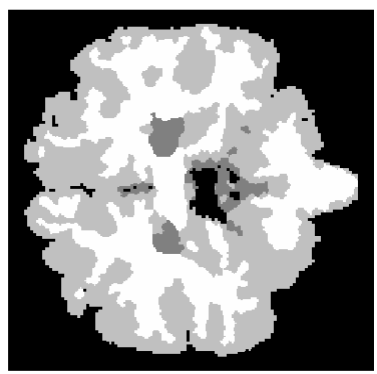

Figure 11. Ensemble

Fig. 12 and Fig. 13 depict the results obtained by application of JS and DSC on 20 training and testing images for six clustering algorithms and the proposed ensemble algorithm for each three classes WM, GM, CSF and the average of classes. 


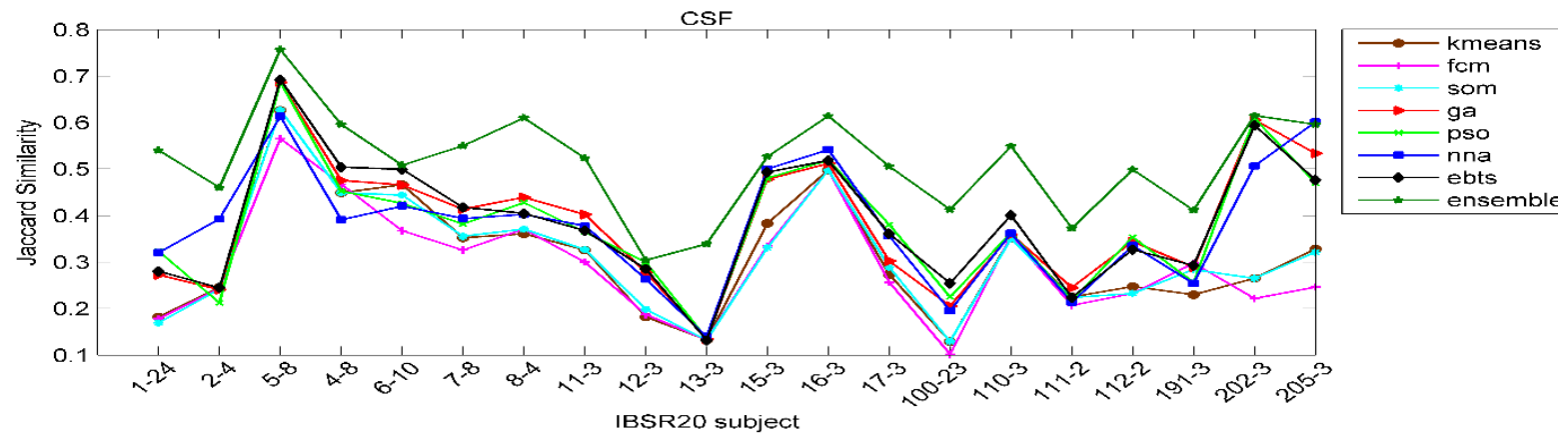

(a)

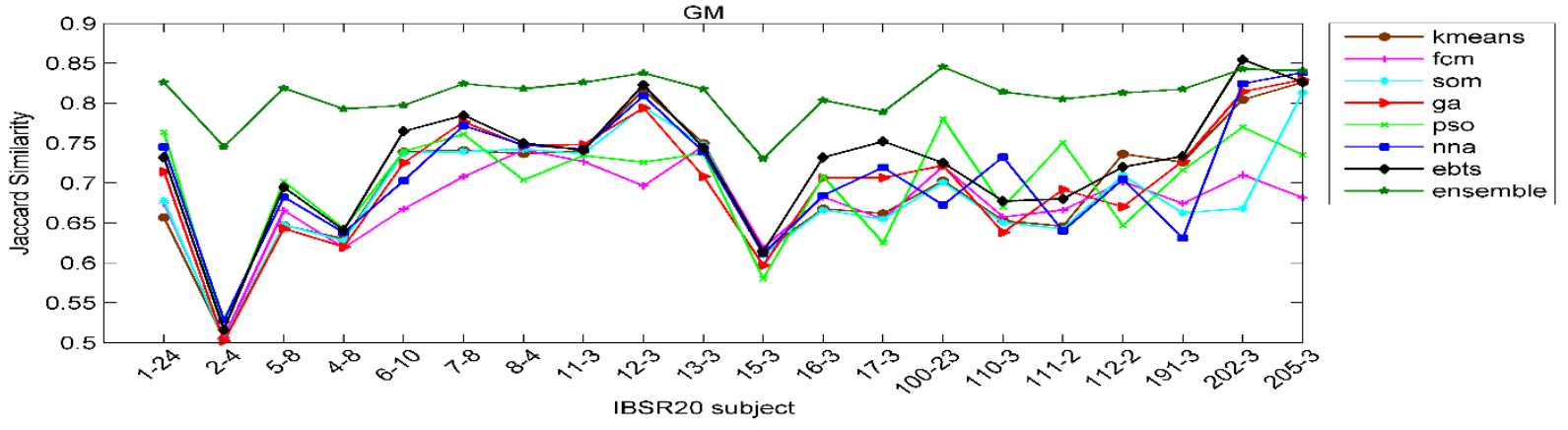

(b)

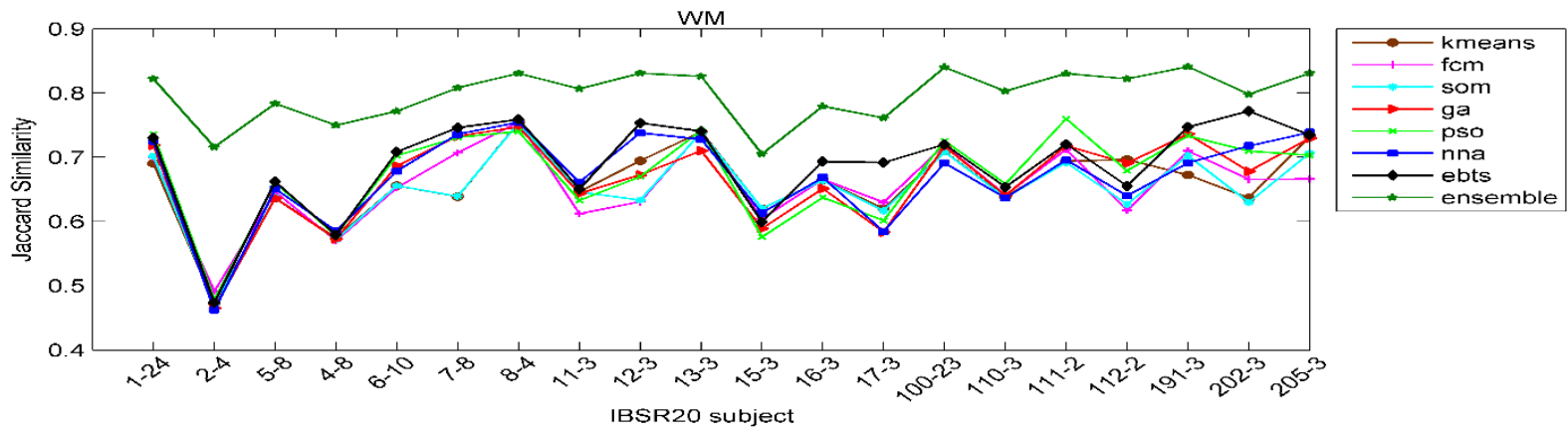

(c)

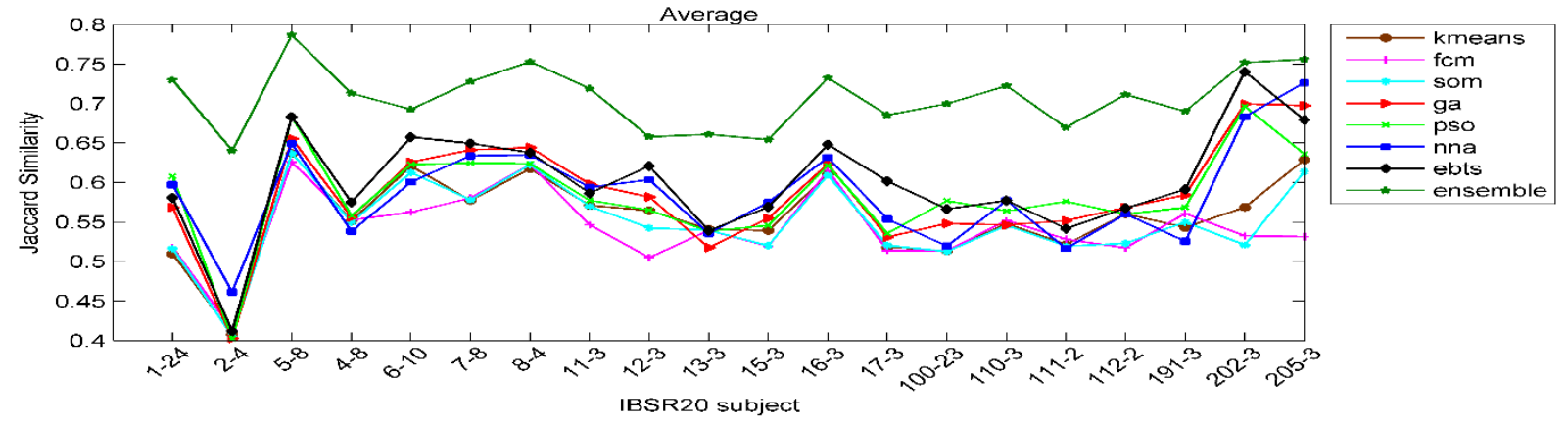


Figure 15. JS criterion results for IBSR20 dataset, slice 20: Error! Reference source not found. CSF, Error! Reference source not found. GM, Error! Reference source not found. WM, Error! Reference source not found.

Average 


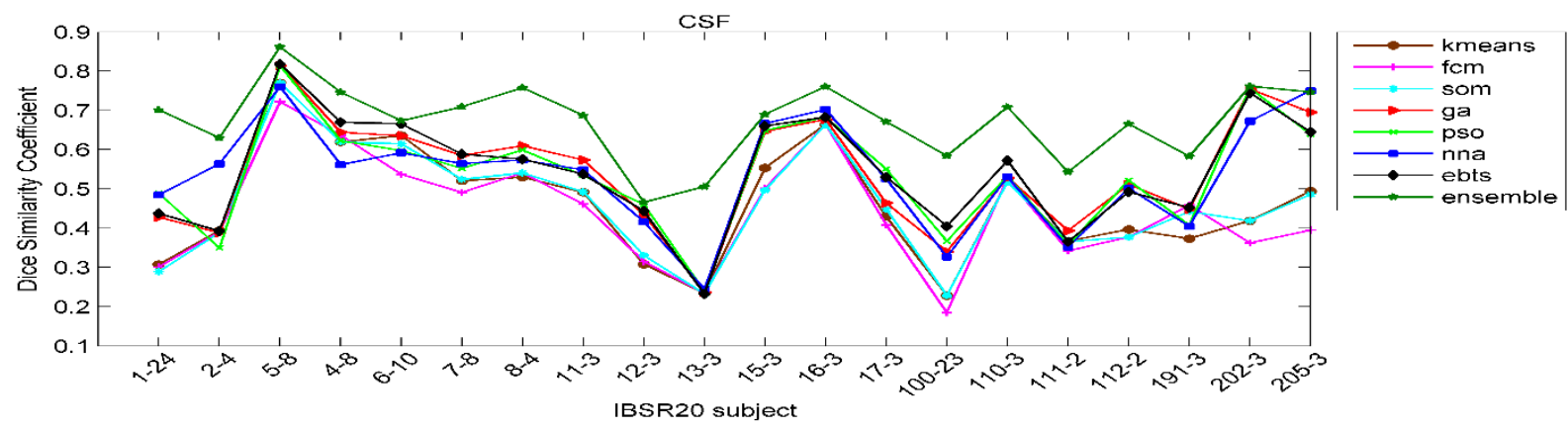

(a)

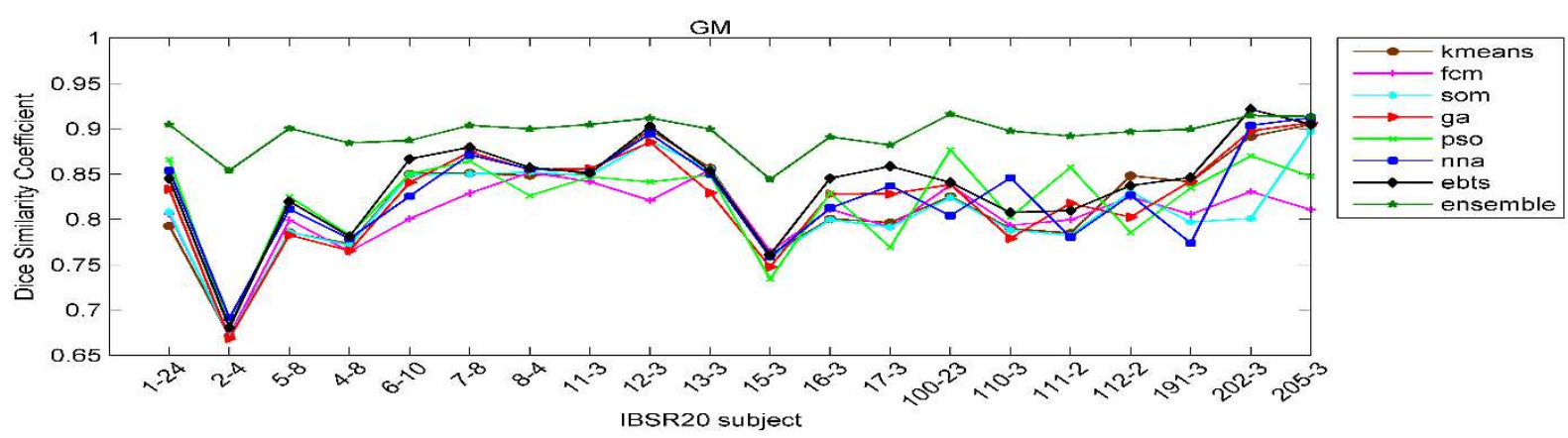

(b)

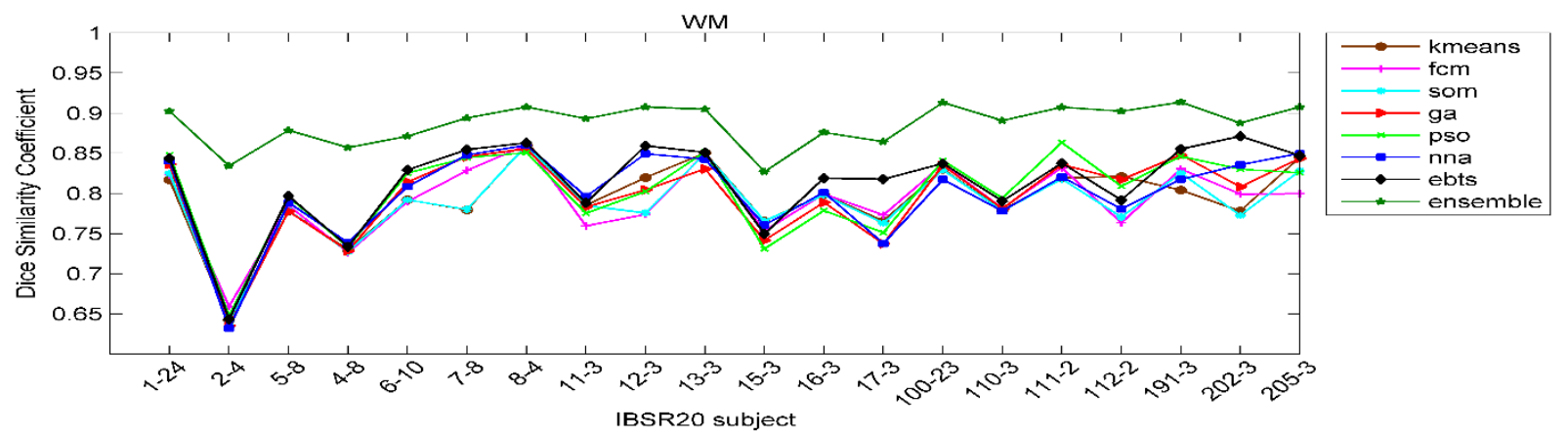

(c)

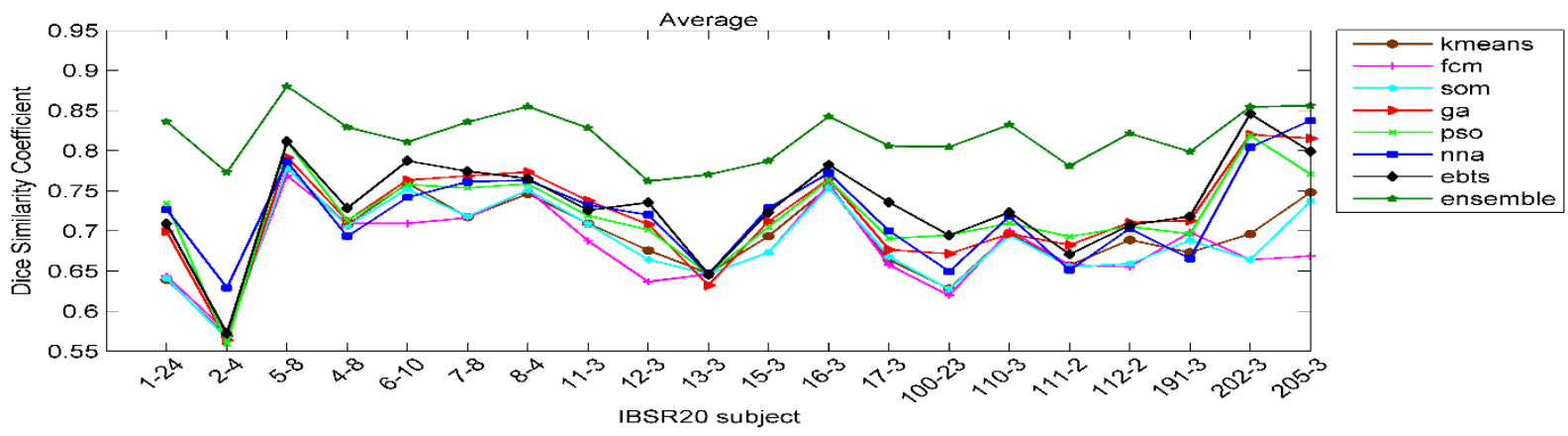

(d)

Figure 13. DSC criterion results for IBSR20 dataset, slice 20: Error! Reference source not found. CSF, Error! Reference source not found. GM, Error! Reference source not found. WM, Error! Reference source not found. Average 


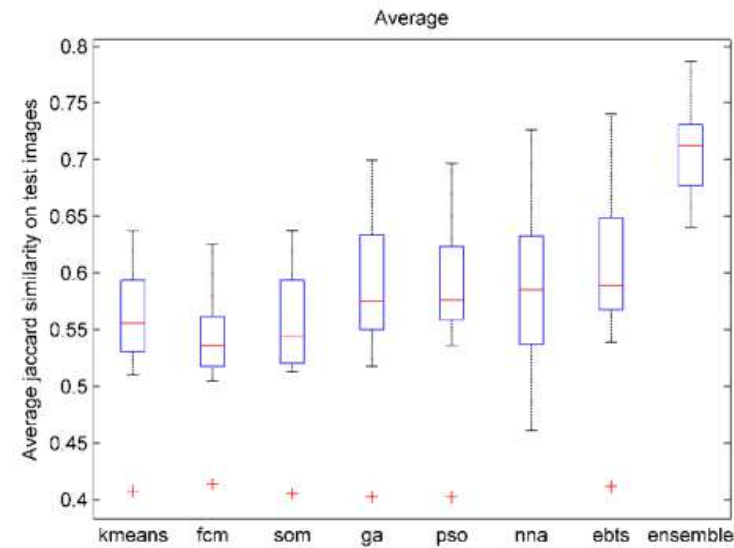

(a)

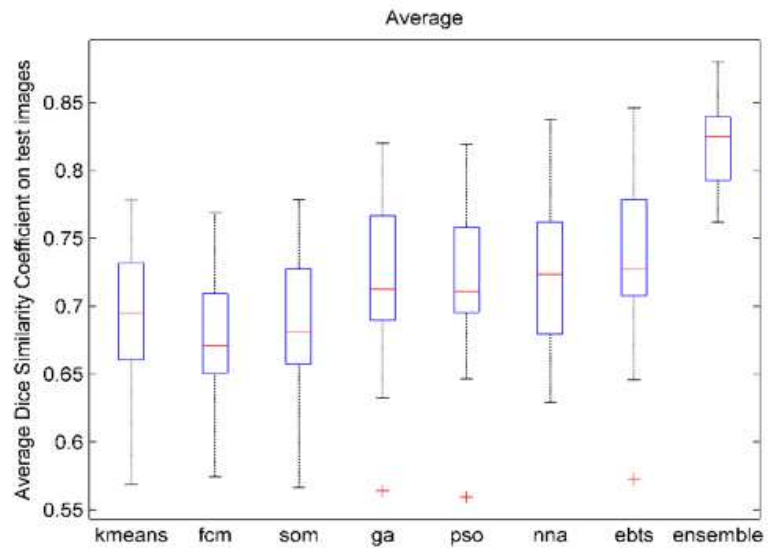

(b)

Figure 14. The box plot of JS and DSC average results for IBSR20 dataset

To get a quality comparison and validate the superiority of the proposed algorithm over the other algorithms, box plots were used here. Fig. 13 illustrate the Box plots of JS and DSC for all of the segmentation methods. The proposed method achieves more accurate results than the other methods and scores the highest median results for all of the tissues.

The Table. 9 shows the accuracy, accuracy, and diagnosis of MRI segmentation images of the brain using the proposed method and other methods.

Table 9. Comparison of the accuracy, precision, recall and error of MRI segmentation images using the proposed method and other methods.

\begin{tabular}{ccccc}
\hline Method & Accuracy\% & Specificity \% & Sensitivity\% & Error\% \\
\hline KNN & 58.95 & $\mathbf{5 8 . 9 5}$ & $\mathbf{1 0 0}$ & 41.04 \\
MLP & 56.74 & 23.42 & 92.30 & 43.25 \\
SVM & 56.35 & 56.35 & $\mathbf{1 0 0}$ & 43.64 \\
My-Approach & $\mathbf{7 3 . 8 8}$ & 45.38 & 56.21 & $\mathbf{2 6 . 1 1}$ \\
\hline
\end{tabular}

As shown in Table. 9, the accuracy of the proposed method with other methods for MRI segmentation is $73.88 \%$, SVM is $56.35 \%$, MLP is $56.74 \%$ and the KNN method is $58.95 \%$. With these interpretations, the improvement in the accuracy of the proposed method compared to the SVM, MLP and KNN methods is $17.53 \%, 17.14 \%$ and $14.93 \%$, respectively. The sensitivity of the proposed method based on geometric features with other methods for MRI segmentation is $56.21 \%$, SVM is $100 \%$, MLP is $92.3 \%$, and $\mathrm{KNN}$ is $100 \%$. The specificity of the proposed method based on geometric features with other methods for MRI segmentation is $45.38 \%$, SVM is $56.35 \%$, MLP is $23.42 \%$ and KNN is $58.95 \%$. With this interpretation, the recovery rate of the proposed method compared to the MLP method is $21.96 \%$. The error rate of the proposed method based on geometric features with other methods for MRI segmentation $26.11 \%$, the SVM method is $43.64 \%$, the MLP is $43.25 \%$, and the KNN method is $41.04 \%$. With these interpretations, the improvement in the error rate of the proposed method is $17.53 \%$, $17.14 \%$ and $14.93 \%$, respectively, compared with the SVM, MLP and KNN methods.

\subsection{Discussion}

As can be seen from the results, the proposed method is based on accurate, precision, recall and error criteria recovery in accordance with other methods. The diagnostic criteria of the proposed method are 
good; But sometimes one of the most important limitations of this paper can be the lack of proper performance for big data and its execution time.

\section{Abbreviation}

\begin{tabular}{|c|c|}
\hline Abbreviation & Meaning \\
\hline MR & Magnetic resonance \\
\hline NNA & neural network algorithm \\
\hline SLIC & simple linear iterative clustering \\
\hline MLP & multi-layer perceptron neural network algorithm \\
\hline SVM & Support vector machine \\
\hline KNN & K nearest neighbor \\
\hline MRI & Magnetic resonance imaging \\
\hline CT & computed tomography \\
\hline WM & white matter \\
\hline GM & gray matter \\
\hline FCM & fuzzy c-means \\
\hline CSF & cerebrospinal fluid \\
\hline FCM & fuzzy c-means \\
\hline SOM & self-organizing mapping \\
\hline GA & genetic algorithm \\
\hline $\mathrm{ABC}$ & artificial bee colony \\
\hline $\mathrm{ACO}$ & ant colony optimization \\
\hline PSO & particle swarm optimization \\
\hline $\mathrm{DE}$ & differential evolution algorithm \\
\hline SOMs & self-organizing mappings \\
\hline ITDS & Information theoretic discriminative segmentation \\
\hline FDNN & fuzzy deep neural network \\
\hline HFS-SOM & histogram based fast segmentation-self-organizing mapping \\
\hline EGS-SOM & $\begin{array}{l}\text { with entropy gradient segmentation- self-organizing } \\
\text { mapping }\end{array}$ \\
\hline DWT & discrete wavelet transform \\
\hline JS & Jacquard similarity \\
\hline DSC & Dice Similarity Coefficient \\
\hline FN & False Negative \\
\hline TN & True Negative \\
\hline
\end{tabular}

\section{Declaration}

\section{Availability of data and materials}

In this study, an image dataset from the Internet Brain Segmentation Database (IBSR) is considered that is prepared at the Massachusetts Hospital Morphometric Analysis Center (http://www.cma.mgh.harvard.edu/ibsr/) .

\section{Competing interests}

The authors declare no competing financial interests

\section{Funding}

The authors no funding for this work .

\section{Authors' contributions}


Miss. Jafari designed and conducted experiments and drafted the manuscript. Dr. Ghaffari conceptualized the project, supervised experimental design, conduct and interpretation of studies, and edited the manuscript.

\section{Acknowledgements}

We thank Miss. Jafari for their technical expertise and their helpful discussions.

\section{Authors' information}

Hamidreza Ghaffari received accordingly his bachelor in Computer Engineering and Master of Software Engineering at Sharif University of Technology in Tehran. He is currently $\mathrm{PhD}$ in Software Engineering at Islamic Azad University of Ferdows, Iran. He has the papers published in national and international conferences and Journals. His research interests include software engineering techniques specifically in the area of data mining, image processing and mixed integer programming. His email address is: hghaffari_papers@ferdowsiau.ac.ir

Fatemeh Jafari received BSc in Computer Engineering, Birjand University of Technology, Iran (2009), MSc in Artificial Intelligence, University of Sistan and Baluchestan, Iran (2016) and She is currently PhD student of Artificial Intelligence and Robotics, Islamic Azad University of Ferdows, Iran. Fields of Interest: Digital Image Processing, esp. for cancer detection. Presenting some conference papers. Her email address is: st.jafari@ferdowsiau.ac.ir

\section{CONCLUSIONS AND SUGGESTIONS}

In this paper, a novel segmentation technique was presented that combines six steps: (1) SLIC algorithm to segment input image to super pixels, (2) three base clustering algorithms such as SOM, FCM, and K-means along with three heuristics GA, PSO and NNA to cluster the clustering output from first stage to three classes, (3) combination (ensemble) of results obtained by clusters, (4) The improved GrowCut method, based on the geometric properties of the images, has been used to segmentation the image of the brain from MRI images. (5) With the help of Zernik's technique, image features were extracted based on the features of the edges and the texture of the images. (6) training a classification ensemble learning model. The number of created super pixels by SLIC algorithm depends on the initial value of super pixels determined by the user. As these algorithms are used for feature extraction, thus the proper selection of initial super pixel size may have an effective role in the performance of the SVM cluster technique. If the size of super pixel is large, the number of extracted features would be less, leading to less computation burden on the algorithm. Of course, this may result in reduced segmentation accuracy. By contrast, small super pixel size leads to increased execution time of the algorithm and likely improved cluster technique accuracy. Thus, a dynamic approach was employed in this paper to determine the initial size of super pixel for SLIC algorithm that in proportion to the input reverse tries to balance computational load and clustering accuracy. Another important point regarding superior performance of the proposed algorithm compared to the other algorithms is to use a relative frequency when a label is attributed to each super pixel. The reason why this approach is employed is to have higher distance between the members of each classes. In particular, CSF class has the smaller size compared to the other two classes. In general, the most noticeable advantage of this approach is to use an ensemble clustering technique to improve segmentations of brain MR tissues and employ a novel metaheuristic NNA along with the other algorithms. The testing stage in this method is more effective and accurate than a sole clustering method. The performance of the proposed method was measured using different evaluation criteria such as JS and DSC. The experiment results demonstrated that this method outperforms the other existing algorithms. 
For the future works, as the proposed algorithm is comprised of four main steps, improvements may be obtained in all four steps, leading to overall performance improvement of the algorithm. For instance, SLIC algorithm may be replaced by super pixel Rate Entropy algorithm to construct super pixel and then evaluate the performance of the method. Thus, significant changes and/or improvements may be employed on feature extraction section that is the most important and effective step for cluster model learning. In the second step, other validated metaheuristics may be employed in this field or the improved version of these six algorithms can be used.

\section{References}

[1] N.M. Portela, G.D. Cavalcanti,T.I. Ren, Semi-supervised clustering for MRI Brain image segmentation. Expert Systems with Applications, 2014. 41(4): p. 1492-1497.

[2] Z. Ji, J. Liu, G. Cao, Q. Sun,Q. Chen, Robust spatially constrained fuzzy c-means algorithm for brain MR image segmentation. Pattern recognition, 2014. 47(7): p. 2454-2466.

[3] E. Abdel-Maksoud, M. Elmogy, R. Al-Awadi, Brain tumor segmentation based on a hybrid clustering technique. Egyptian Informatics Journal, 2015. 16(1): p. 71-81.

[4] Z. Ji, Y. Xia, Q. Sun, Q. Chen,D. Feng, Adaptive scale fuzzy local Gaussian mixture model for brain MR image segmentation. Neurocomputing, 2014. 134: p. 60-69.

[5] A. Tiwari, S. Srivastava,M. Pant, Brain Tumor Segmentation and Classification from Magnetic Resonance Images: Review of selected methods from 2014 to 2019. Pattern Recognition Letters, 2019.

[6] E.-S.A. El-Dahshan, H.M. Mohsen, K. Revett,A.-B.M. Salem, Computer-aided diagnosis of human brain tumor through MRI: A survey and a new algorithm. Expert systems with Applications, 2014. 41(11): p. 5526-5545.

[7] A. Wadhwa, A. Bhardwaj,V.S. Verma, A review on brain tumor segmentation of MRI images. Magnetic resonance imaging, 2019.

[8] P. Dhanalakshmi,T. Kanimozhi, Automatic segmentation of brain tumor using K-Means clustering and its area calculation. International Journal of advanced electrical Electronics Engineering, 2013. 2(2): p. 130-134.

[9] C. Singh,A. Bala, A local Zernike moment-based unbiased nonlocal means fuzzy C-Means algorithm for segmentation of brain magnetic resonance images. Expert Systems with Applications, 2019. 118: p. 625-639.

[10] S. Manikandan, K. Ramar, M.W. Iruthayarajan,K. Srinivasagan, Multilevel thresholding for segmentation of medical brain images using real coded genetic algorithm. Measurement, 2014. 47: p. 558-568.

[11] S. De, S. Bhattacharyya,P. Dutta, Automatic magnetic resonance image segmentation by fuzzy intercluster hostility index based genetic algorithm: An application. Applied Soft Computing, 2016. 47: p. 669-683.

[12]D. Karaboga,C. Ozturk, A novel clustering approach: Artificial Bee Colony (ABC) algorithm. Applied soft computing, 2011.11(1): p. 652-657.

[13] B. Akay, A study on particle swarm optimization and artificial bee colony algorithms for multilevel thresholding. Applied Soft Computing, 2013. 13(6): p. 3066-3091.

[14]H. Gao, Z. Fu, C.-M. Pun, H. Hu, R. Lan, A multi-level thresholding image segmentation based on an improved artificial bee colony algorithm. Computers Electrical Engineering, 2018. 70: p. 931-938.

[15] A.E. Hassanien, H.M. Moftah, A.T. Azar,M. Shoman, MRI breast cancer diagnosis hybrid approach using adaptive ant-based segmentation and multilayer perceptron neural networks classifier. Applied Soft Computing, 2014. 14: p. 62-71.

[16] H.R. Shahdoosti,Z. Tabatabaei, MRI and PET/SPECT image fusion at feature level using ant colony based segmentation. Biomedical Signal Processing Control, 2019. 47: p. 63-74.

[17] T.X. Pham, P. Siarry,H. Oulhadj, Integrating fuzzy entropy clustering with an improved PSO for MRI brain image segmentation. Applied Soft Computing, 2018. 65: p. 230-242. 
[18] O. Tarkhaneh,H. Shen, An adaptive differential evolution algorithm to optimal multi-level thresholding for MRI brain image segmentation. Expert Systems with Applications, 2019. 138: p. 112820.

[19] A. Sadollah, H. Sayyaadi, A. Yadav, A dynamic metaheuristic optimization model inspired by biological nervous systems: Neural network algorithm. Applied Soft Computing, 2018. 71: p. 747-782.

[20] Y. Kong, Y. Deng, Q. Dai, Discriminative clustering and feature selection for brain MRI segmentation. IEEE Signal Processing Letters, 2014. 22(5): p. 573-577.

[21] Y. Deng, Z. Ren, Y. Kong, F. Bao,Q. Dai, A hierarchical fused fuzzy deep neural network for data classification. IEEE Transactions on Fuzzy Systems, 2016. 25(4): p. 1006-1012.

[22] A. Ortiz, J. Górriz, J. Ramírez, D. Salas-Gonzalez,J.M. Llamas-Elvira, Two fullyunsupervised methods for MRI Brain image segmentation using SOM-based strategies. Applied Soft Computing, 2013. 13(5): p. 2668-2682.

[23] N. Noreen, K. Hayat, S.A. Madani, MRI segmentation through wavelets and fuzzy C-means. World Applied Sciences Journal, 2011. 13:p. 34-39.

[24] R. Katyal, S. Paneri,M. Kuse, Gaussian intensity model with neighborhood cues for fluid tissue categorization of multisequence MRI Brain images. Proceedings of the MICCAI Grand Challenge on MRI Brain Image Segmentation, 2013.

[25] H. Al-Dmour, A. Al-Ani, A clustering fusion technique for MRI Brain tissue segmentation. Neurocomputing, 2018. 275: p. 546-559. 
TABLE 1 (A). The JS results obtained by application of the algorithms on training dataset

\begin{tabular}{ccccccccccccc}
\hline \multicolumn{3}{c}{ K-means } & \multicolumn{3}{c}{ FCM } & \multicolumn{3}{c}{ SOM } & \multicolumn{3}{c}{ EBTs } \\
\hline$C S F$ & $G M$ & $W M$ & $C S F$ & $G M$ & $W M$ & $C S F$ & $G M$ & $W M$ & $C S F$ & $G M$ & $W M$ \\
\hline 0.1820 & 0.6567 & 0.6906 & 0.1775 & 0.6749 & 0.6997 & 0.1692 & 0.6779 & 0.7018 & 0.2801 & 0.7319 & 0.7300 \\
\hline 0.2453 & 0.5051 & 0.4726 & 0.2430 & 0.5062 & 0.4914 & 0.2416 & 0.5036 & 0.4706 & 0.2445 & 0.5161 & 0.4741 \\
\hline 0.6264 & 0.6472 & 0.6367 & 0.5652 & 0.6656 & 0.6459 & 0.6264 & 0.6476 & 0.6373 & 0.6924 & 0.6947 & 0.6623 \\
\hline 0.4489 & 0.6299 & 0.5742 & 0.4674 & 0.6189 & 0.5700 & 0.4489 & 0.6277 & 0.5718 & 0.5040 & 0.6408 & 0.5792 \\
\hline 0.4665 & 0.7392 & 0.6556 & 0.3672 & 0.6676 & 0.6527 & 0.4439 & 0.7379 & 0.6556 & 0.4993 & 0.7648 & 0.7084 \\
\hline
\end{tabular}

TABLE 1 (B). The JS results obtained by application of the algorithms on training dataset

\begin{tabular}{cccccccccccc}
\hline & GA & & \multicolumn{3}{c}{ PSO } & & & NNA & \multicolumn{3}{c}{ Ensemble Clustering } \\
\hline$C S F$ & $G M$ & $W M$ & $C S F$ & $G M$ & $W M$ & $C S F$ & $G M$ & $W M$ & $C S F$ & $G M$ & $W M$ \\
\hline 0.2724 & 0.7142 & 0.7187 & 0.3236 & 0.7634 & 0.7360 & 0.3201 & 0.7452 & 0.7251 & 0.5404 & 0.8259 & 0.8220 \\
\hline 0.2409 & 0.5021 & 0.4651 & 0.2123 & 0.5156 & 0.4784 & 0.3927 & 0.5282 & 0.4621 & 0.4601 & 0.7453 & 0.7158 \\
\hline 0.6864 & 0.6429 & 0.6362 & 0.6864 & 0.7017 & 0.6602 & 0.6129 & 0.6824 & 0.6508 & 0.7570 & 0.8188 & 0.7835 \\
\hline 0.4754 & 0.6200 & 0.5729 & 0.4524 & 0.6430 & 0.5785 & 0.3909 & 0.6378 & 0.5854 & 0.5956 & 0.7928 & 0.7499 \\
\hline 0.4658 & 0.7252 & 0.6865 & 0.4260 & 0.7396 & 0.7026 & 0.4202 & 0.7029 & 0.6784 & 0.5082 & 0.7973 & 0.7720 \\
\hline
\end{tabular}


Tables 4-7 provide the average results for evaluation criteria of JS and DSC by application of the aforementioned segmentation algorithms on training and testing dataset for WM, GM, and CSF classes. The spatial overlap metric has no impact on estimation of the overall performance in terms of error. Thus, the obtained values of sensitivity, specificity and accuracy are presented for each method in Table 8 . The quantitative results demonstrated that the proposed method outperforms the other base clustering techniques for WM, GM and CSF tissues.

TABLE 2. JS values for segmentation algorithms for training and testing IBSR20 dataset

\begin{tabular}{lllll}
\hline class & k-means & FCM & SOM & EBTs \\
\hline CSF & 0.3127 & 0.2944 & 0.3118 & 0.3884 \\
\hline GM & 0.7000 & 0.6760 & 0.6867 & 0.7253 \\
\hline WM & 0.6593 & 0.6567 & 0.6525 & 0.6895 \\
\hline Average & 0.5573 & 0.5424 & 0.5503 & 0.6011
\end{tabular}

TABLE 3. JS values for segmentation algorithms for training and testing dataset

\begin{tabular}{cccccc}
\hline Dataset & class & GA & PSO & NNA & Ensemble Clustering \\
\hline IBSR20 & CSF & 0.3884 & 0.3791 & 0.3789 & 0.5145 \\
\hline & GM & 0.7041 & 0.7003 & 0.7082 & 0.8103 \\
\hline WM & 0.6660 & 0.6727 & 0.6698 & 0.7978 \\
\hline & Average & 0.5847 & 0.5841 & 0.5857 & 0.7075
\end{tabular}

TABLE 4. DSC values for segmentation algorithms for training and testing dataset

\begin{tabular}{llllll}
\hline Dataset & class & k-means & FCM & SOM & EBTs \\
\hline IBSR20 & CSF & 0.4631 & 0.4427 & 0.4626 & 0.5459 \\
\hline & GM & 0.8212 & 0.8055 & 0.8123 & 0.8385 \\
\hline & WM & 0.7930 & 0.7911 & 0.7880 & 0.8140 \\
\hline & Average & 0.6924 & 0.6798 & 0.6876 & 0.7328
\end{tabular}

TABLE 5. DSC values for segmentation algorithms for training and testing dataset

\begin{tabular}{cccccc}
\hline Dataset & class & GA & PSO & NNA & Ensemble Clustering \\
\hline IBSR20 & CSF & 0.5407 & 0.5363 & 0.5373 & 0.6729 \\
\hline & GM & 0.8239 & 0.8219 & 0.8269 & 0.8949 \\
\hline WM & 0.7973 & 0.8021 & 0.8001 & 0.8870 \\
\hline & Average & 0.7206 & 0.7201 & 0.7215 & 0.8183 \\
\hline
\end{tabular}


TABLE 6. The average segmentation accuracy of algorithms on training and testing dataset

\begin{tabular}{cccc}
\hline Algorithms & Sensitivity & Specificity & Accuracy \\
\hline k-means & 0.9340 & 0.9602 & 0.8354 \\
\hline FCM & 0.9264 & 0.9550 & 0.8185 \\
SOM & 0.9230 & 0.9526 & 0.8113 \\
\hline EBTs & 0.9209 & 0.9506 & 0.8092 \\
\hline GA & 0.9220 & 0.9517 & 0.8100 \\
\hline PSO & 0.9213 & 0.9511 & 0.8090 \\
\hline NNA & 0.9205 & 0.9504 & 0.8080 \\
\hline Ensemble & 0.9519 & 0.9726 & 0.8737 \\
\hline
\end{tabular}

Table 7. Comparison of the accuracy, precision, recall and error of MRI segmentation images using the proposed method and other methods.

\begin{tabular}{ccccc}
\hline Method & Accuracy\% & Specificity\% & Sensitivity\% & Error\% \\
\hline KNN & 58.95 & $\mathbf{5 8 . 9 5}$ & $\mathbf{1 0 0}$ & 41.04 \\
MLP & 56.74 & 23.42 & 92.30 & 43.25 \\
SVM & 56.35 & 56.35 & $\mathbf{1 0 0}$ & 43.64 \\
My-Approach & $\mathbf{7 3 . 8 8}$ & 45.38 & 56.21 & $\mathbf{2 6 . 1 1}$ \\
\hline
\end{tabular}




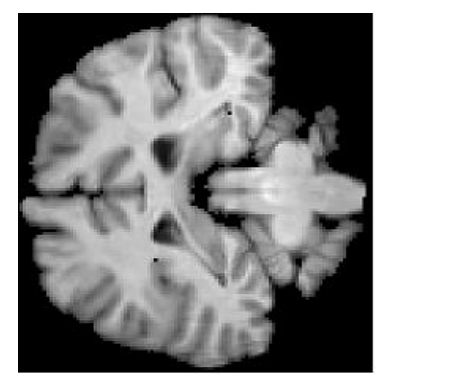

Figure

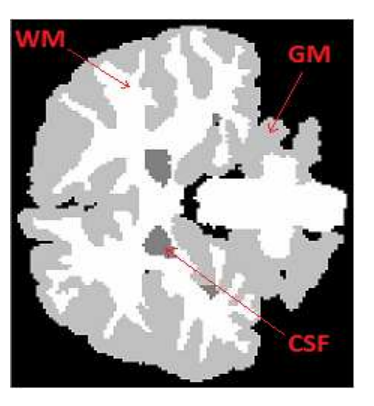

Figure 2. Segmented Image

\begin{abstract}
Figure 2. Segmented Inage
\end{abstract}

(1)

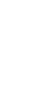




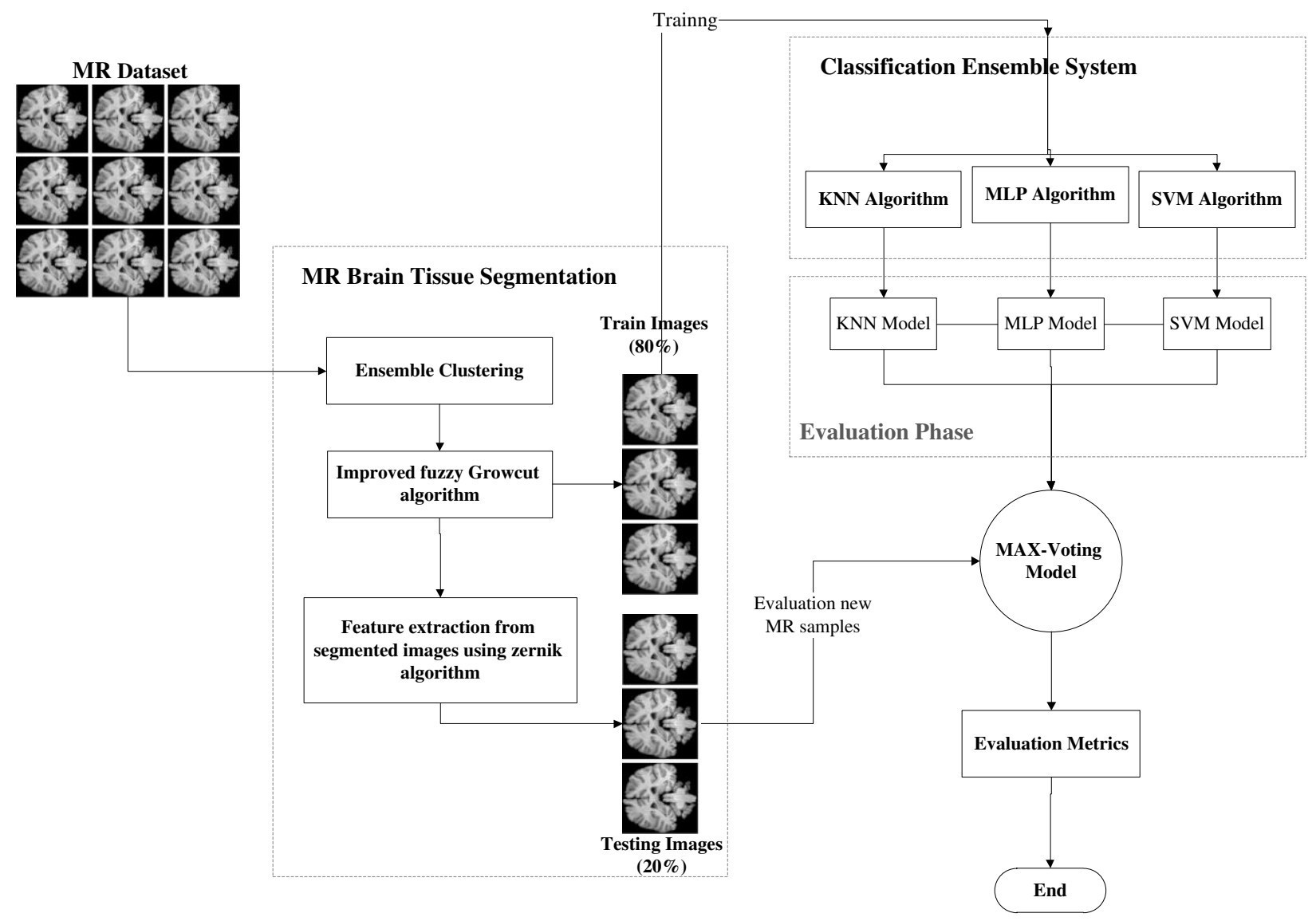

Figure 1. The proposed Architecture 


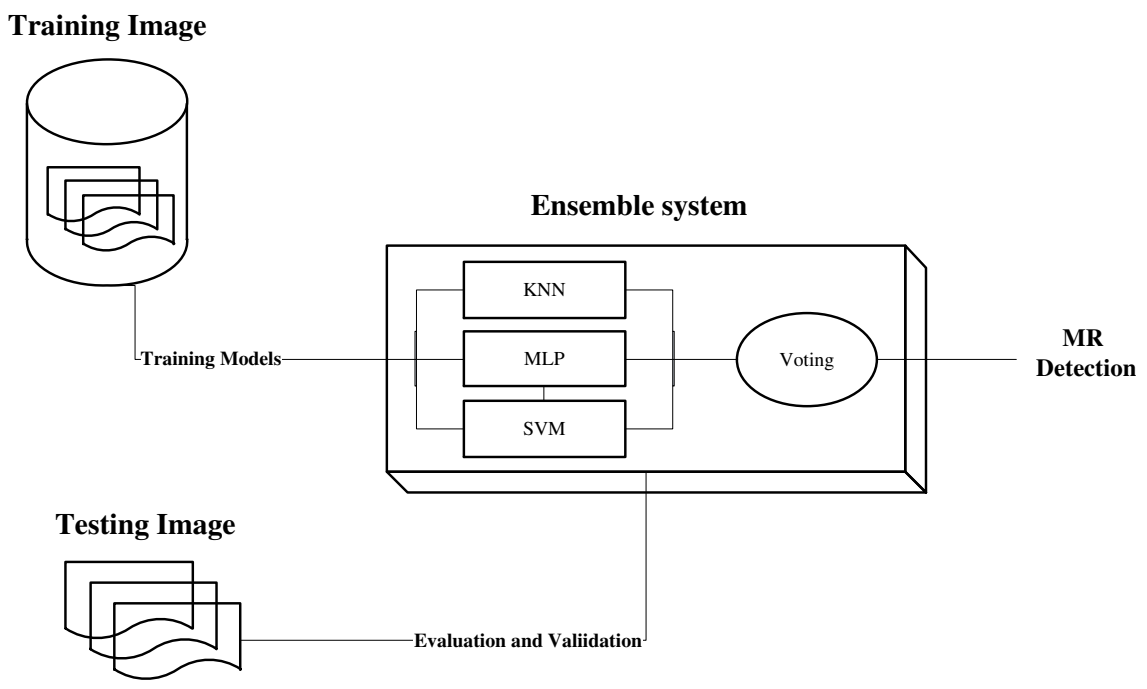

Figure 2. Flowchart of ensemble system for MRI Brain images.

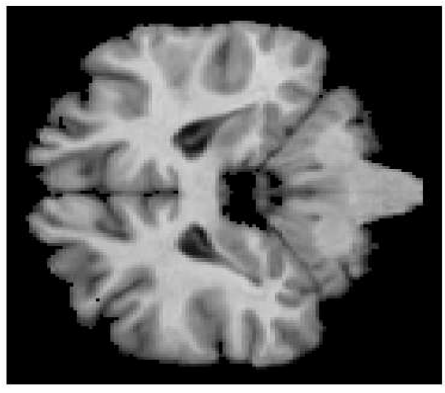

Figure 3. Test image

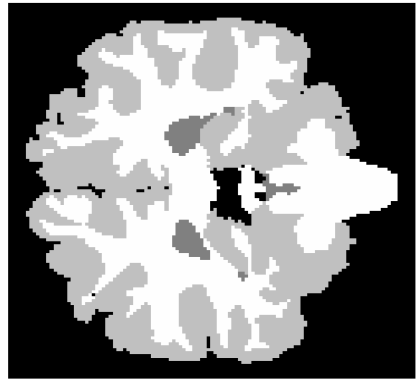

Figure 4. Ground truth

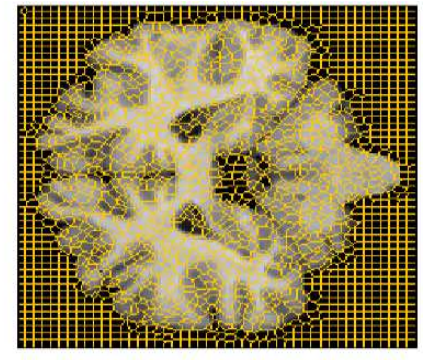

Figure 5. SLIC

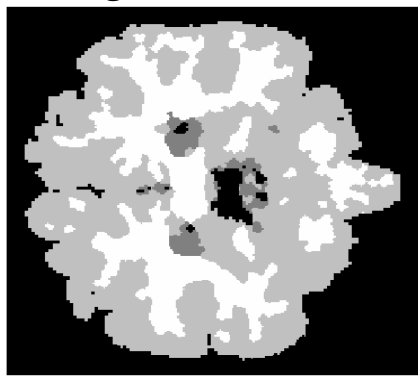

Figure 6. K-means

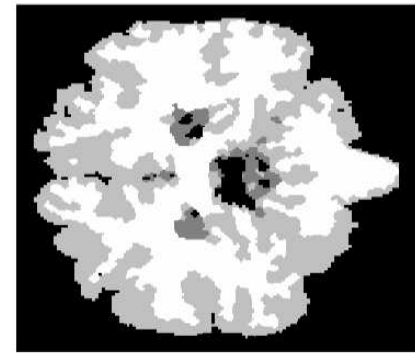

Figure 7. FCM

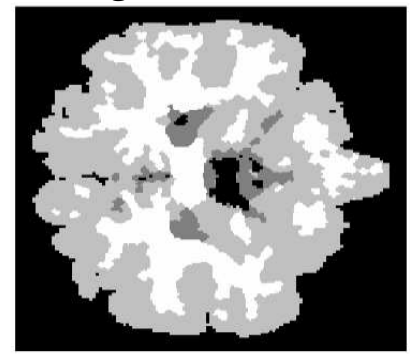

Figure 8. SOM 


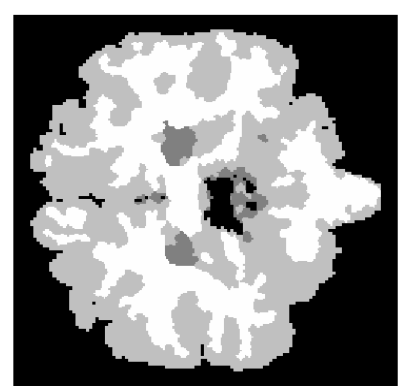

Figure 9. GA

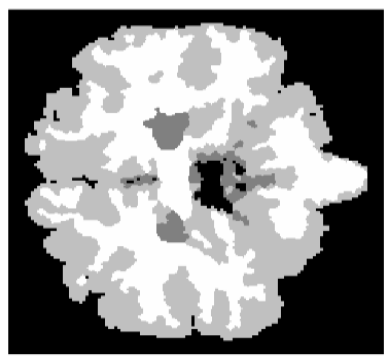

Figure 13. NNA

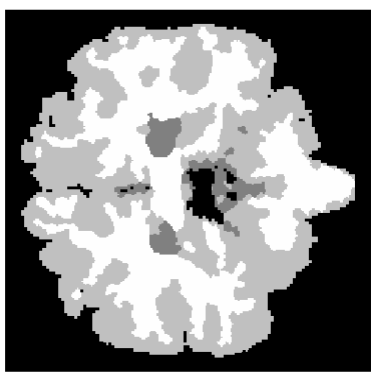

Figure 11. Ensemble clustering

Fig. 12 and Fig. 13 depict the results obtained by application of JS and DSC on 20 training and testing images for six clustering algorithms and the proposed ensemble algorithm for each three classes WM, GM, CSF and the average of classes.

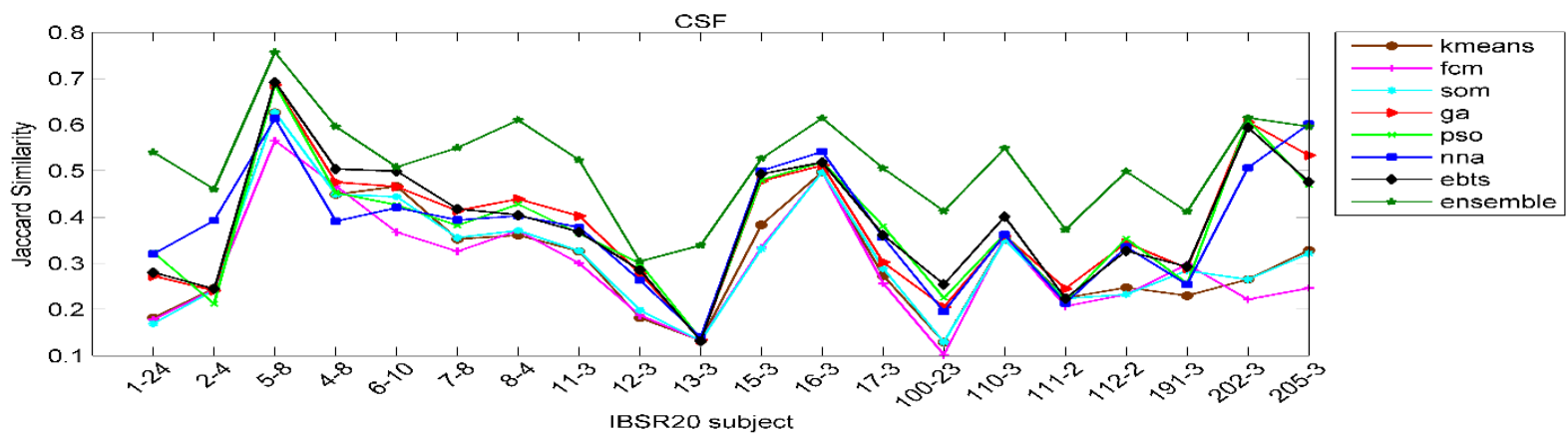

(a)

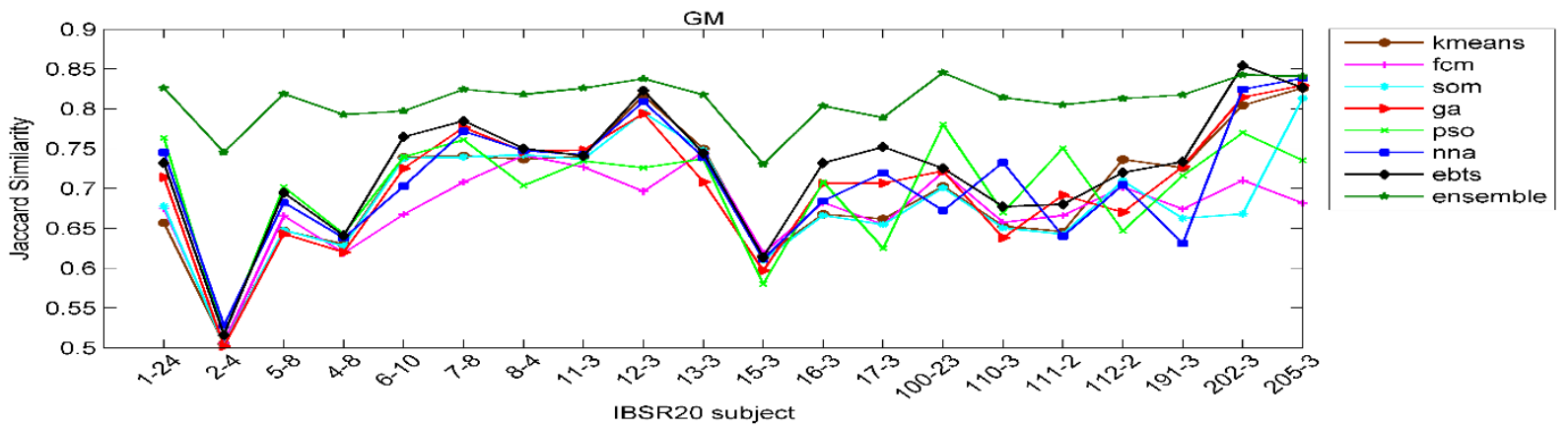

(b)

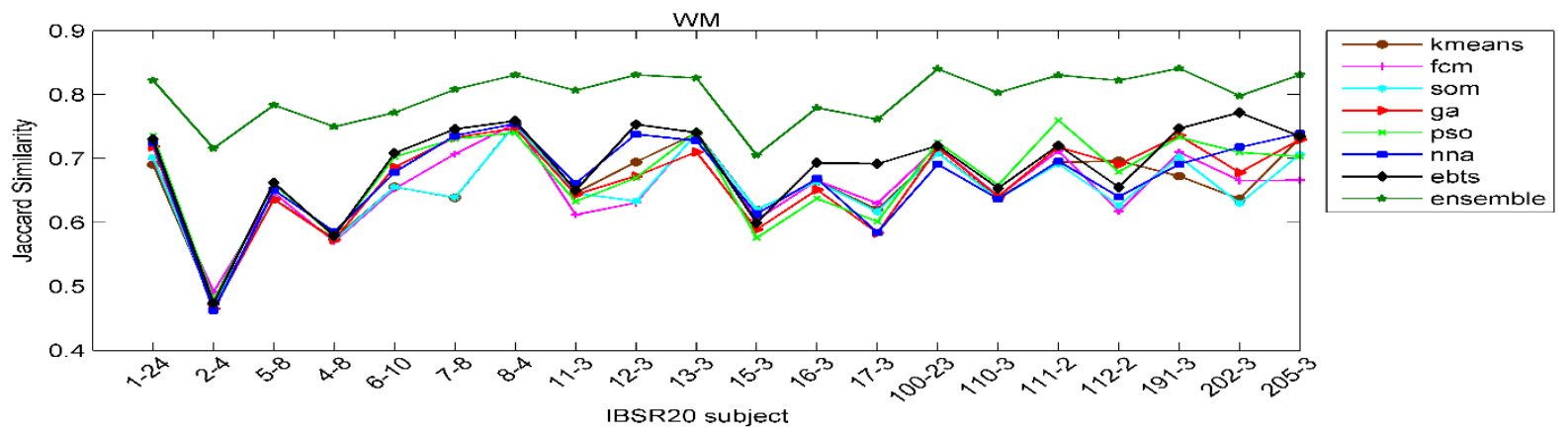


(c)

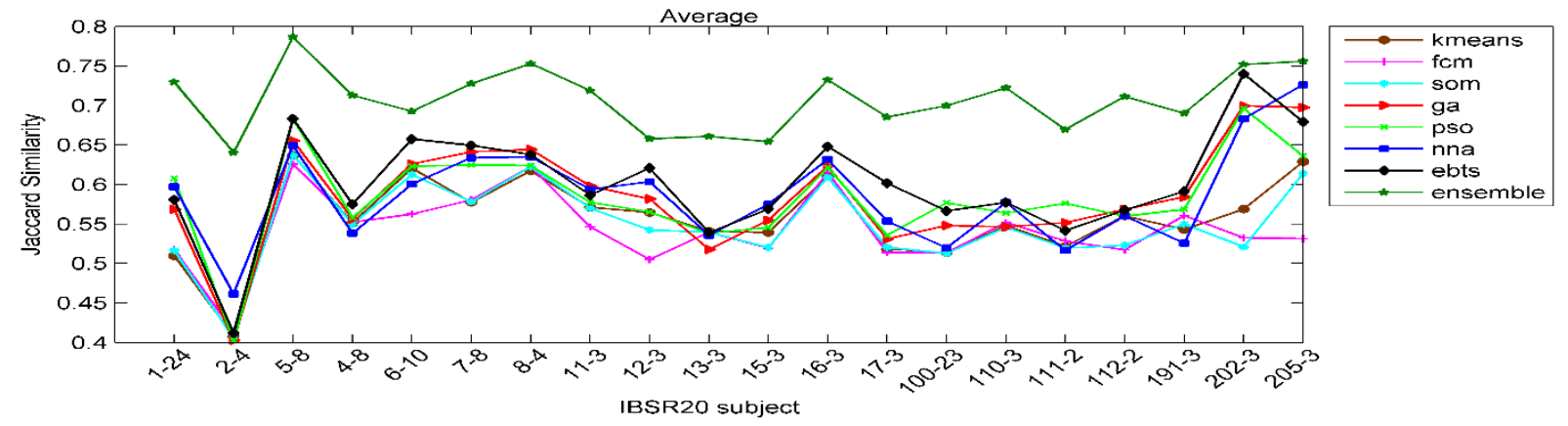

(d)

Figure 15. JS criterion results for IBSR20 dataset, slice 20: Error! Reference source not found. CSF, Error! Reference source not found. GM, Error! Reference source not found. WM, Error! Reference source not found. Average

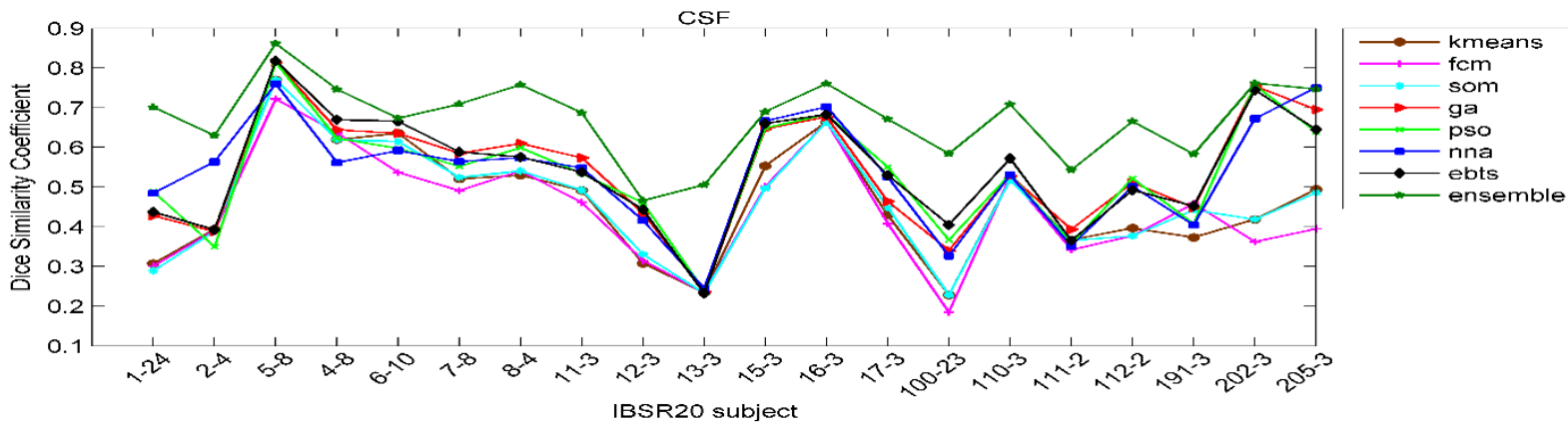


(a)

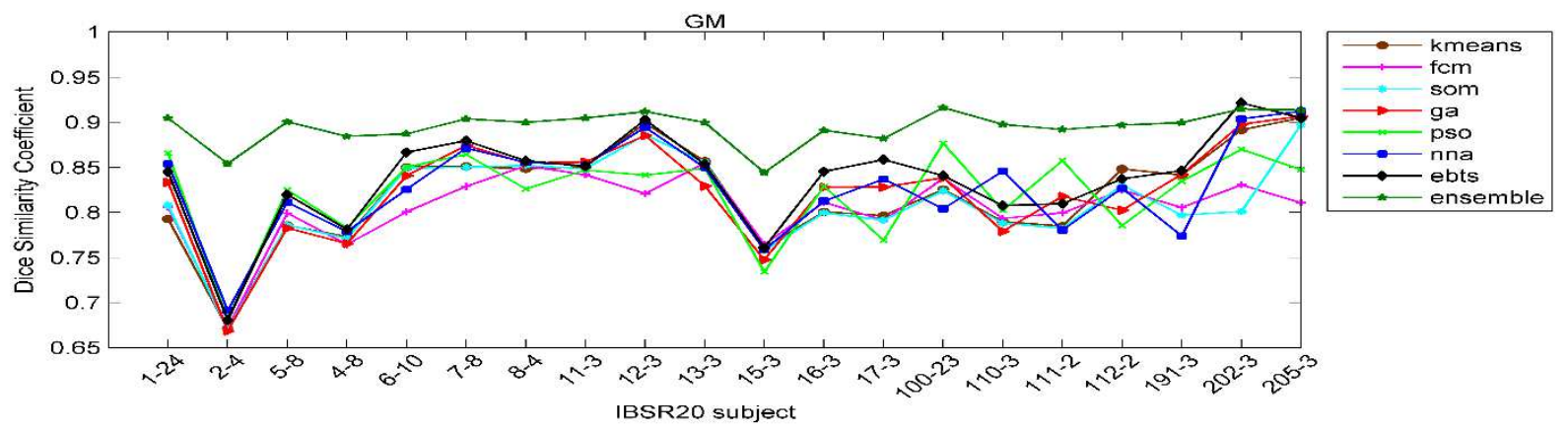

(b)

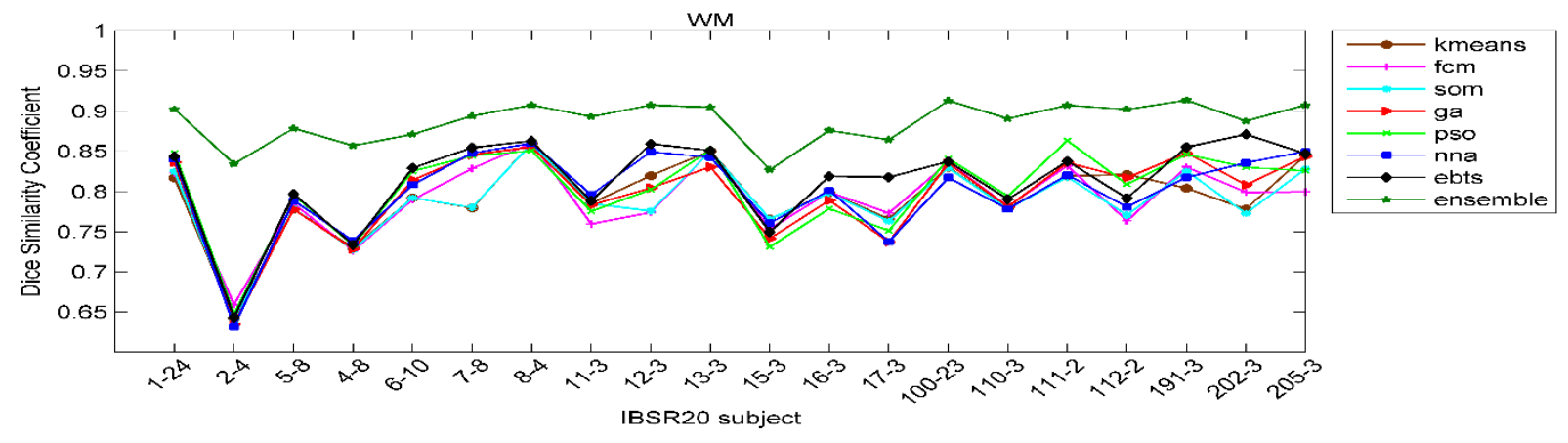

(c)

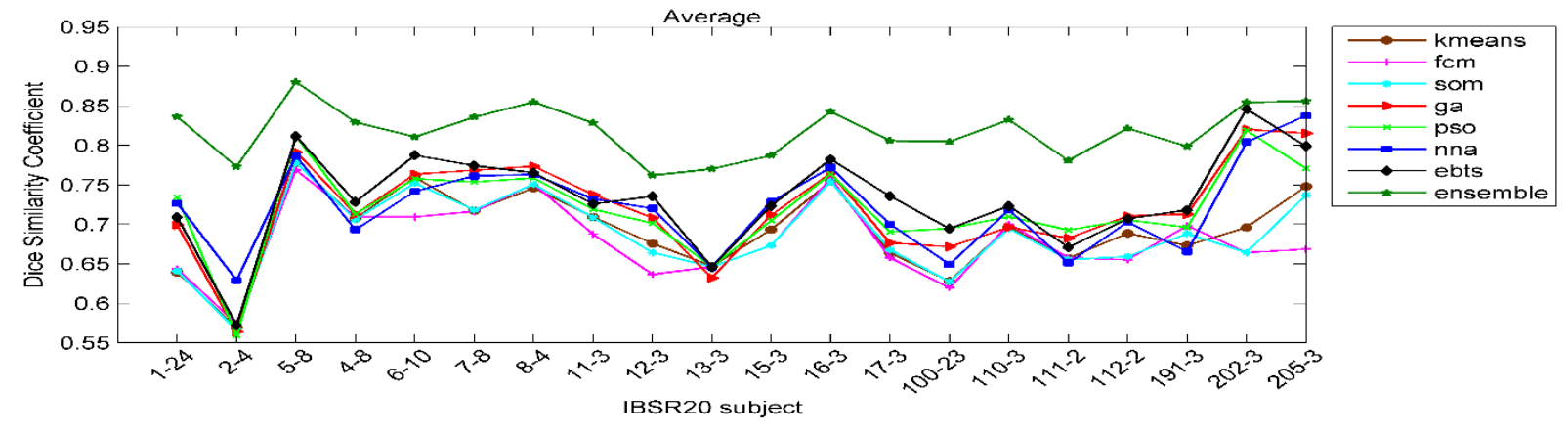

(d)

Figure 13. DSC criterion results for IBSR20 dataset, slice 20: Error! Reference source not found. CSF, Error! Reference source not found. GM, Error! Reference source not found. WM, Error! Reference source not found. Average 


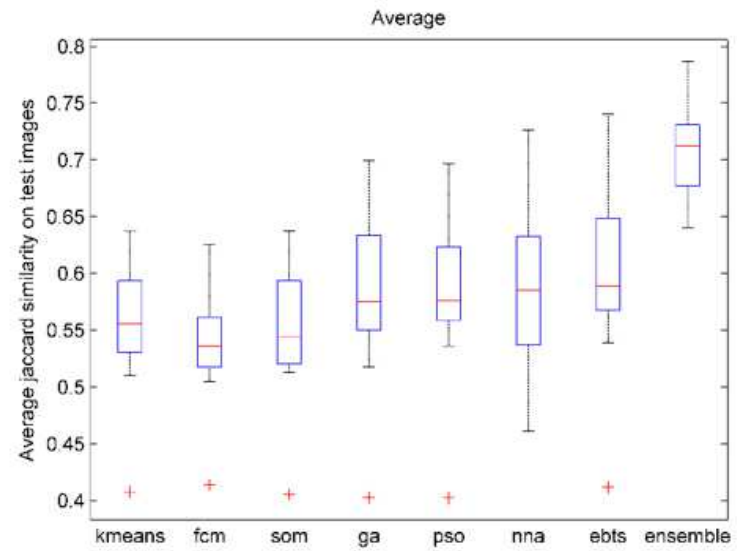

(a)

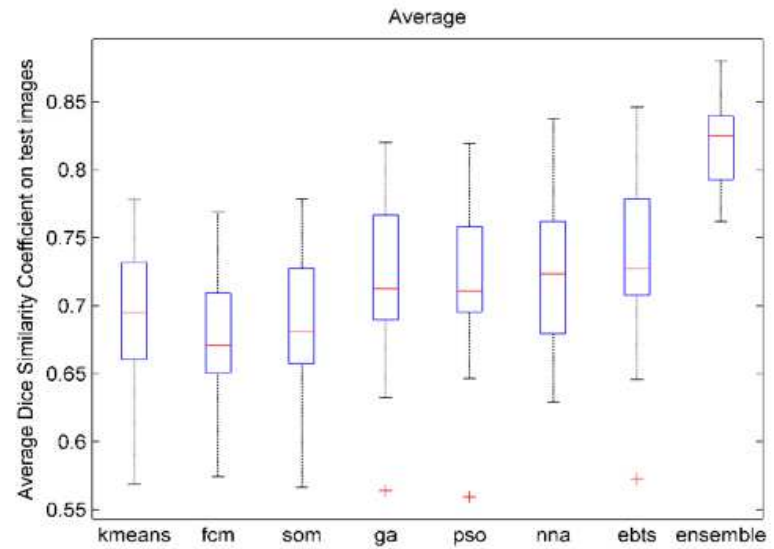

(b)

Figure 14. The box plot of JS and DSC average results for IBSR20 dataset 
Figures

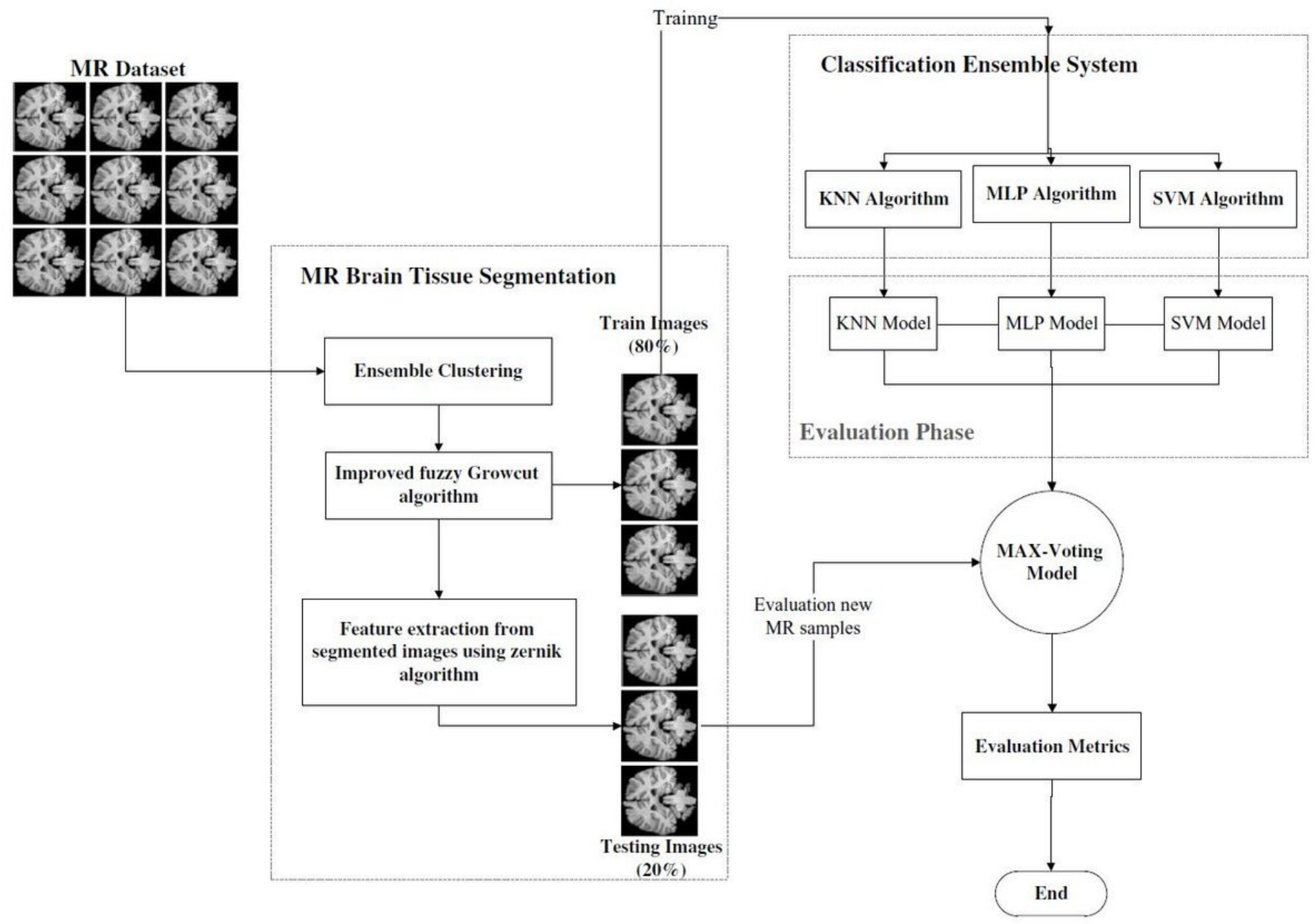

Figure 1

The proposed Architecture 


\section{Training Image}

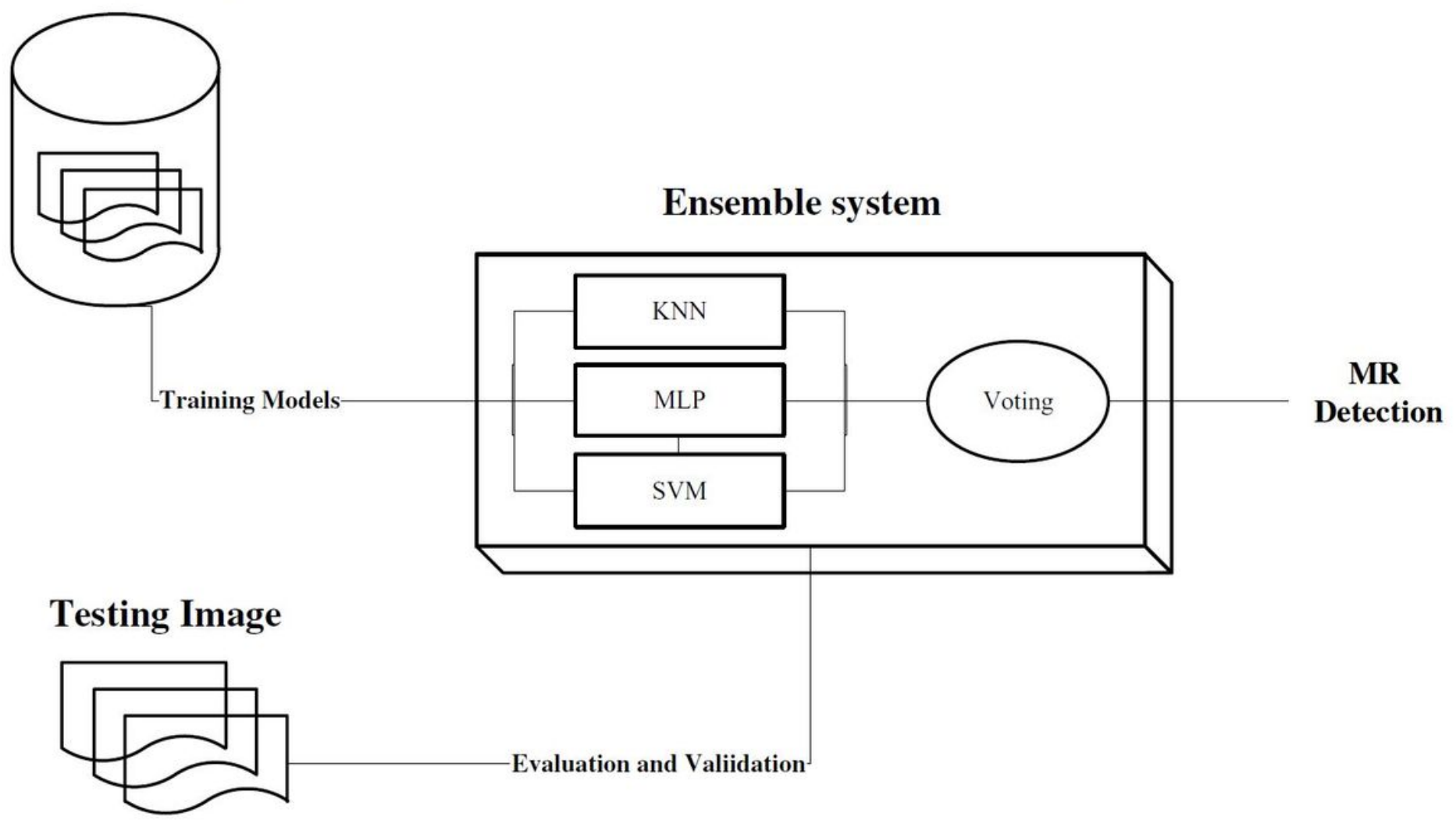

Figure 2

Flowchart of ensemble system for MRI Brain images.

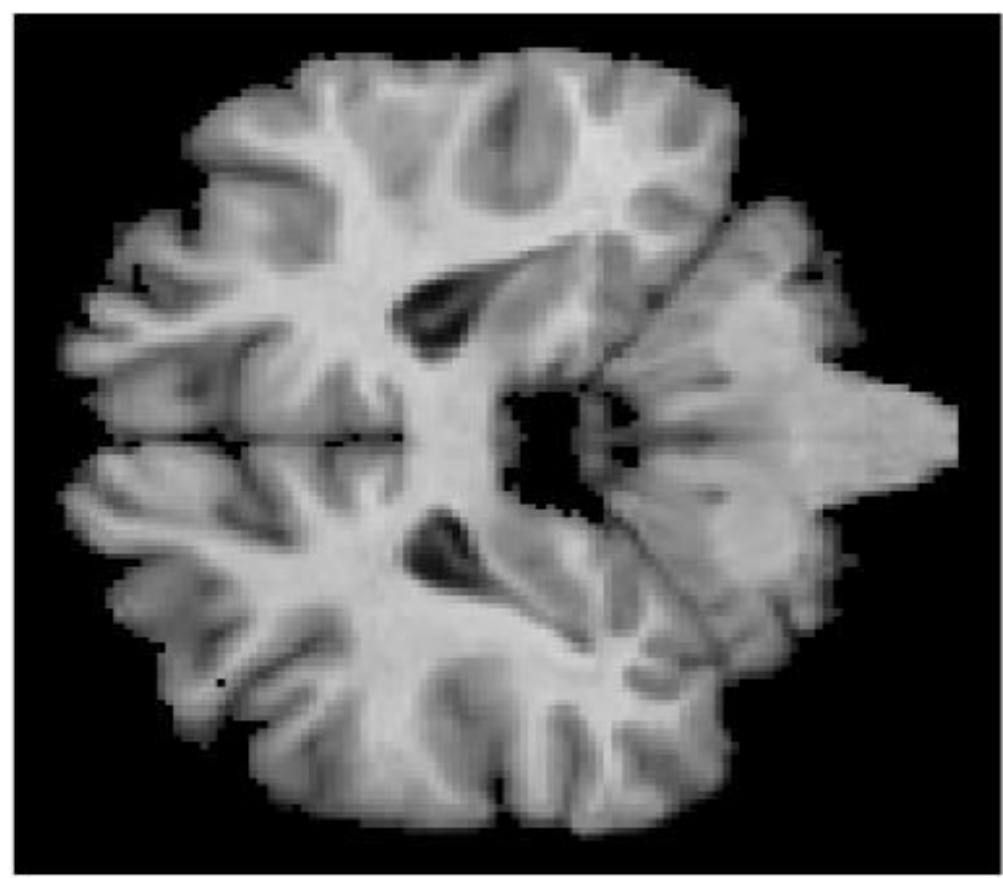

Figure 3 
Test image

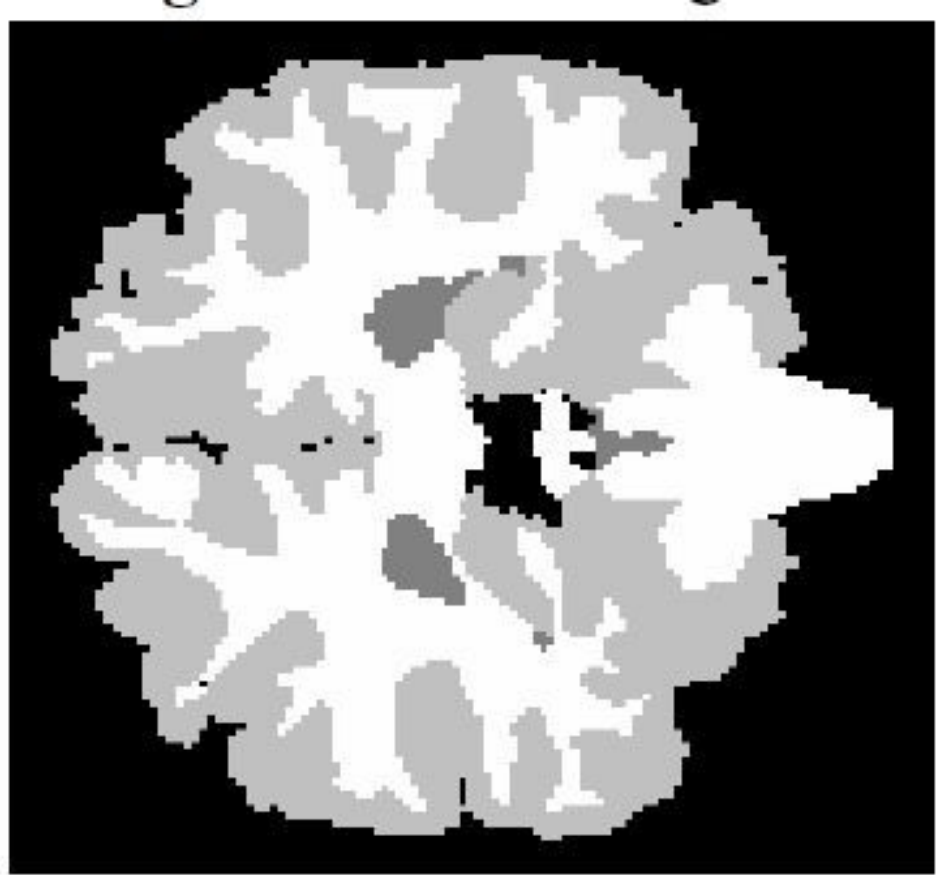

Figure 4

Ground truth

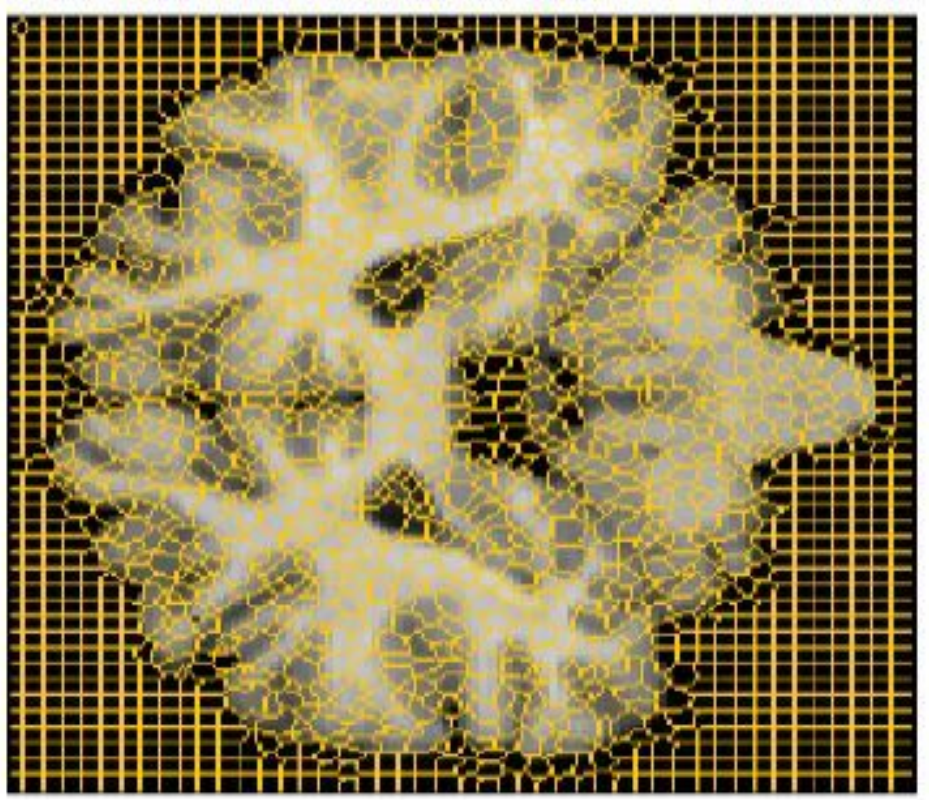

Figure 5

SLIC 


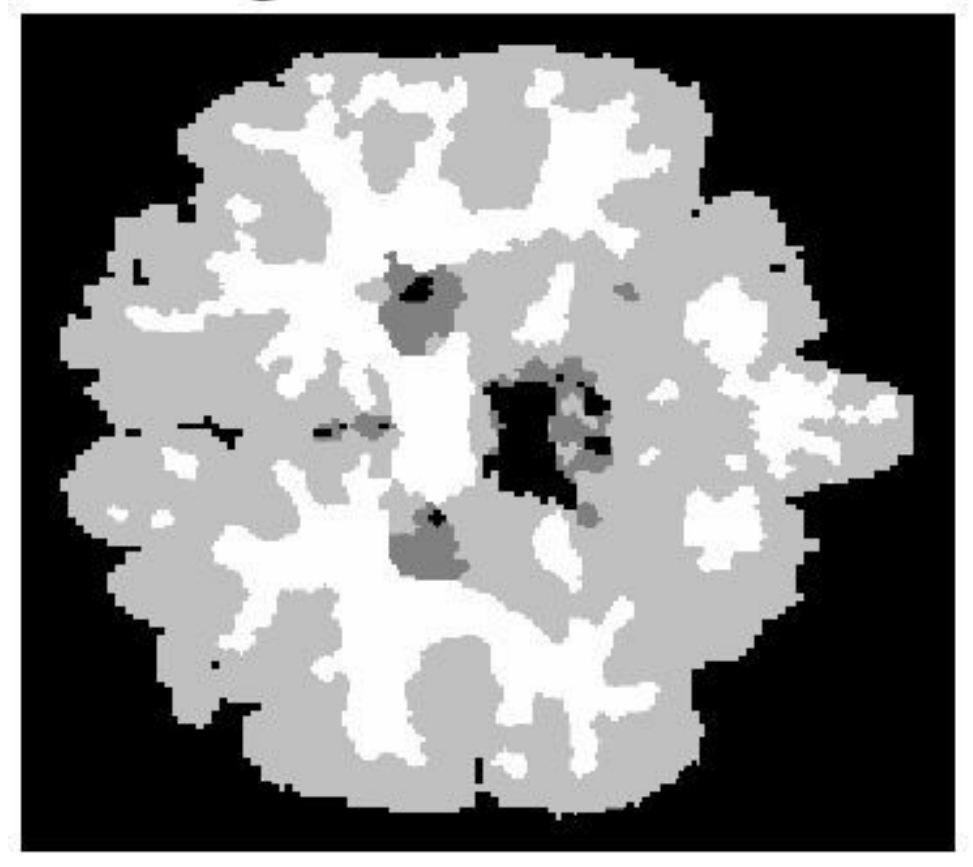

Figure 6

K-means

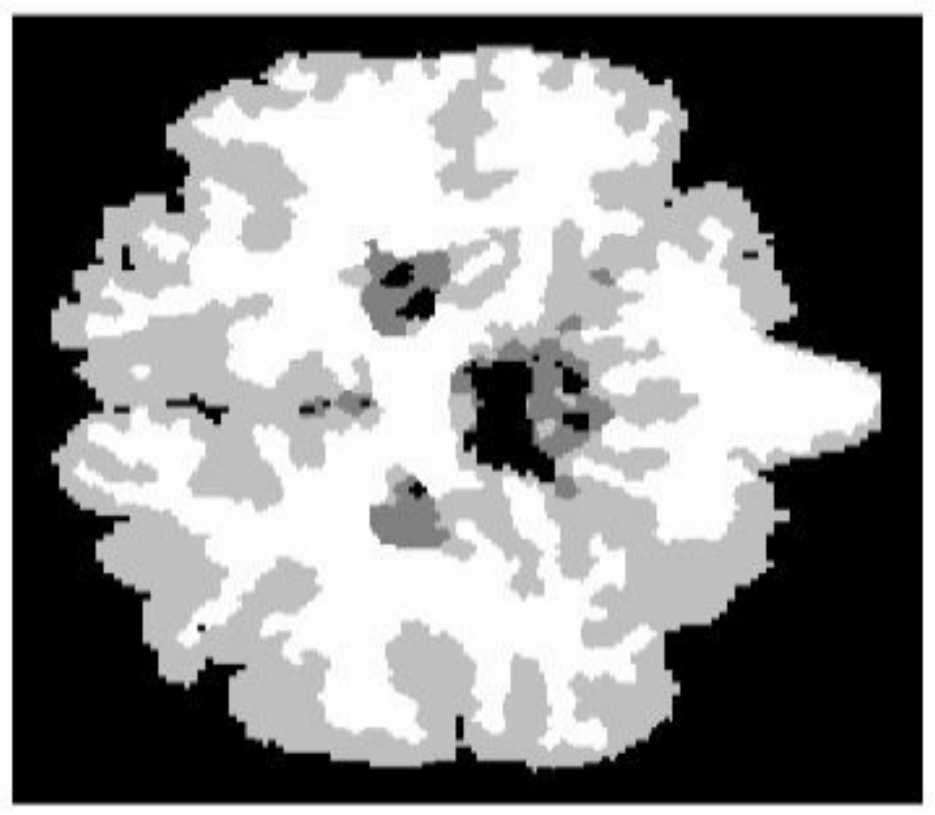

Figure 7

FCM 


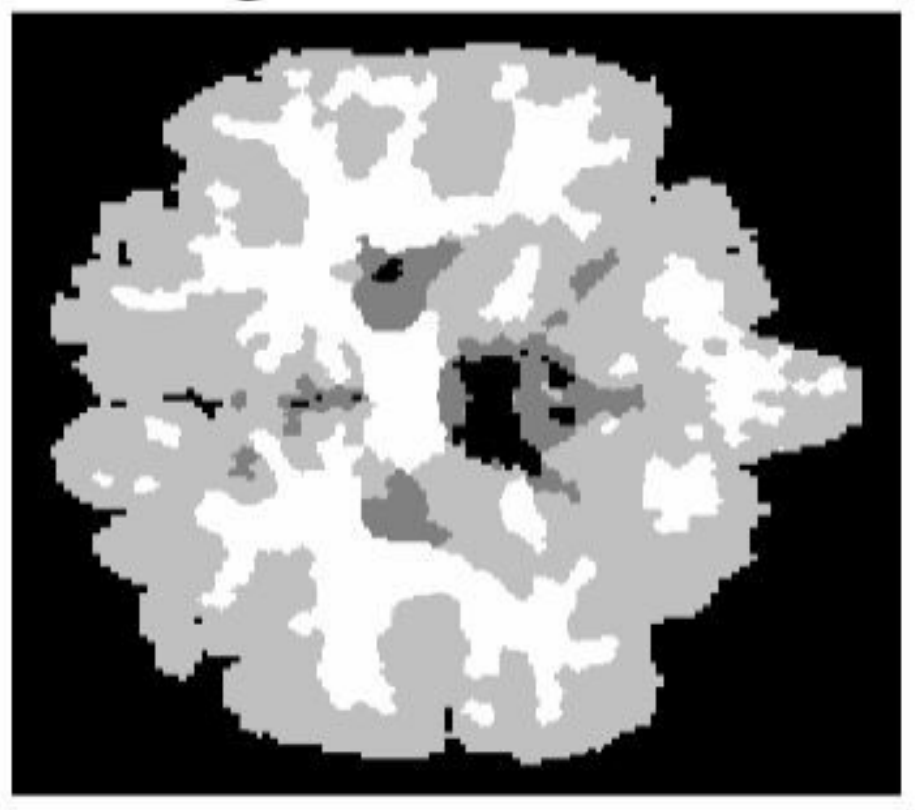

Figure 8

SOM

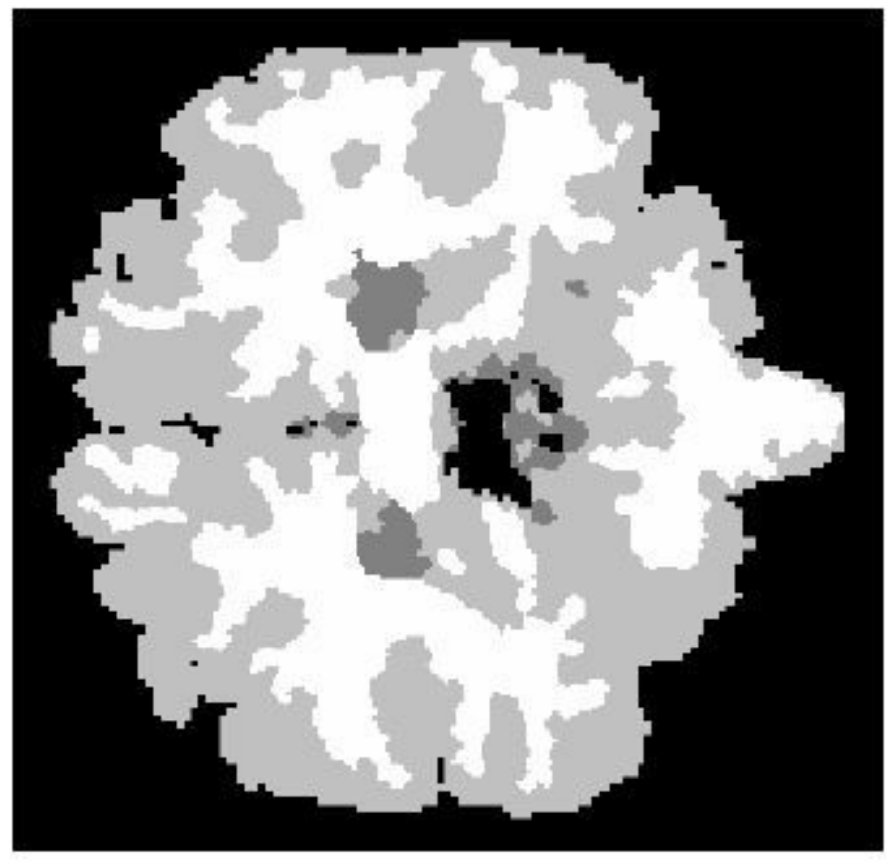

Figure 9

GA 


\section{Image not available with this version}

Figure 10

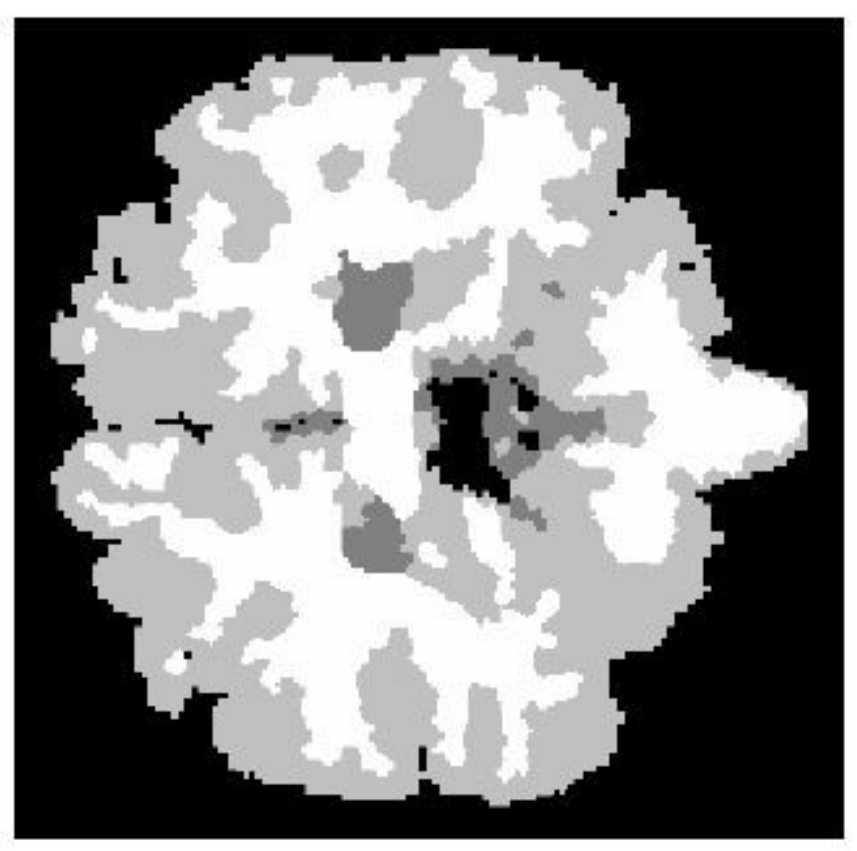

Figure 11

Ensemble clustering

Image not available with this version

Figure 12 


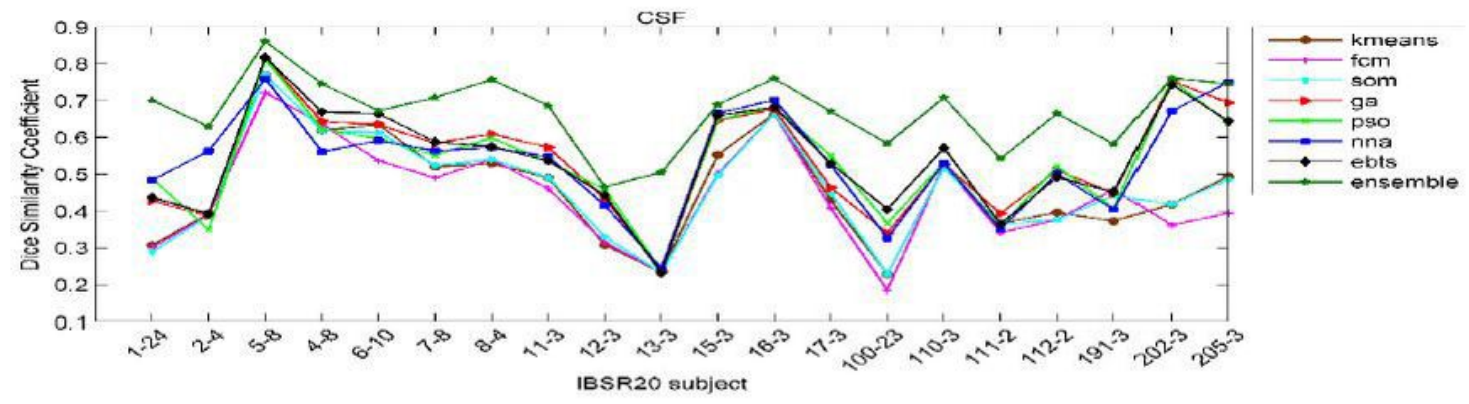

(a)

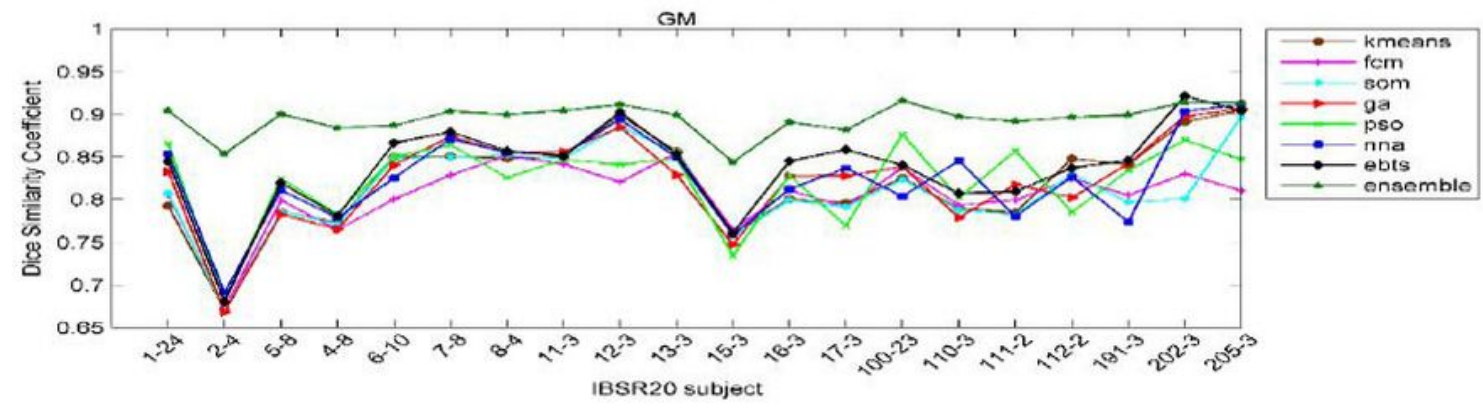

(b)

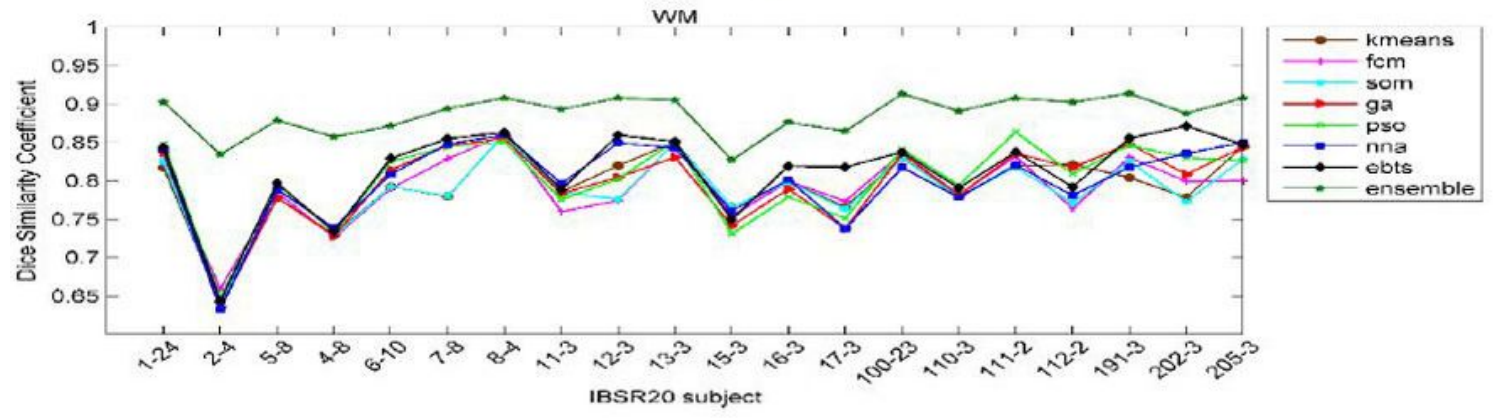

(c)

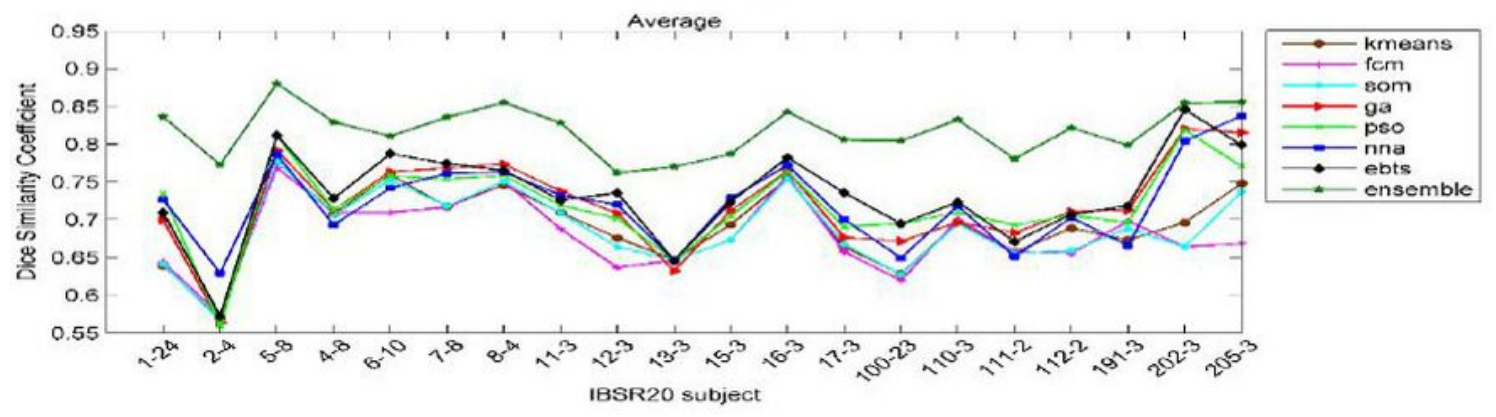

(d)

\section{Figure 13}

DSC criterion results for IBSR20 dataset, slice 20: Error! Reference source not found. CSF, Error! Reference source not found. GM, Error! Reference source not found. WM, Error! Reference source not found. Average 


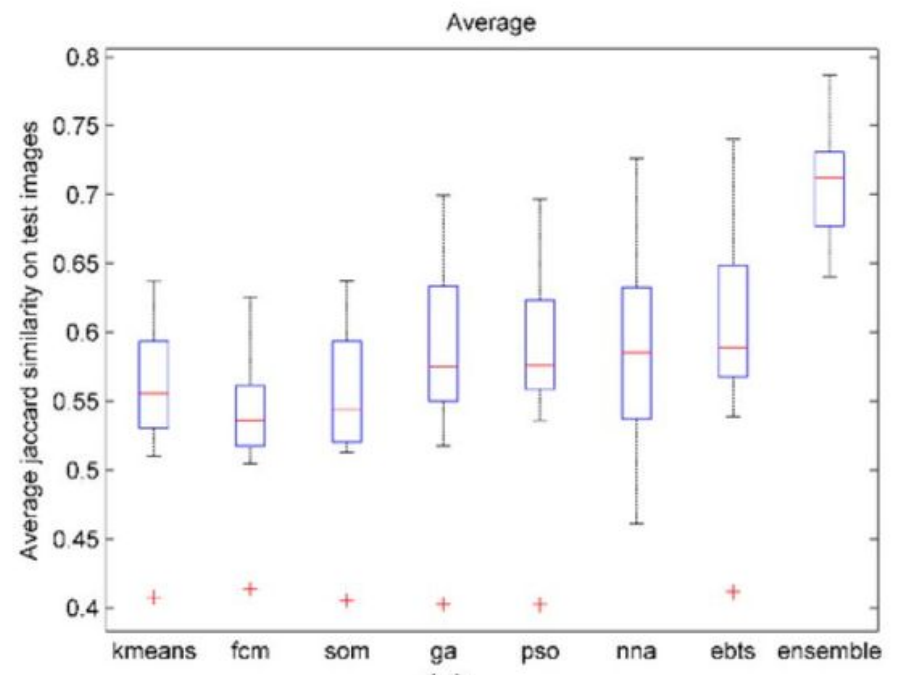

(a)

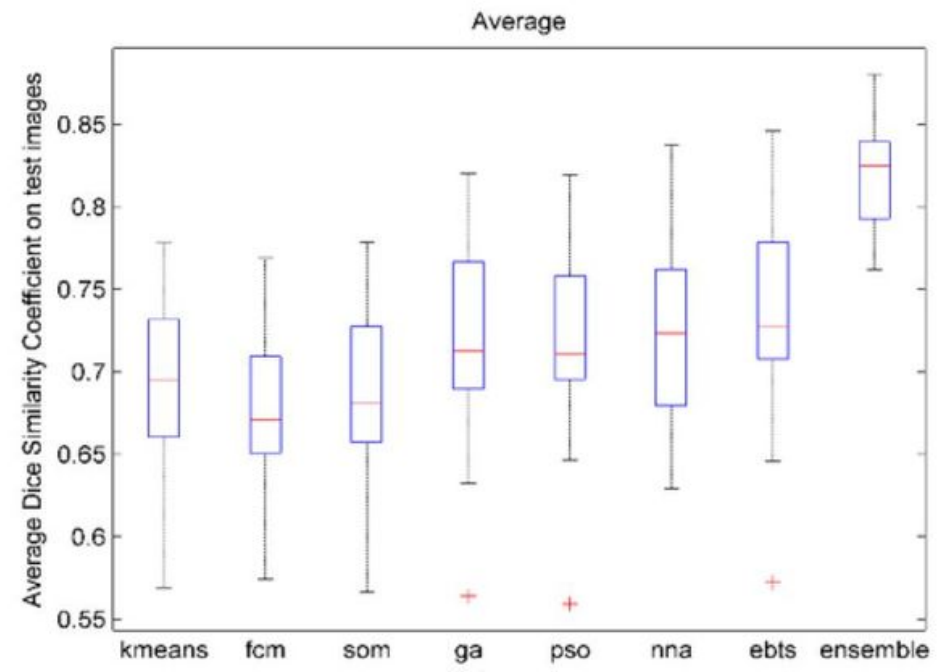

(b)

Figure 14

The box plot of JS and DSC average results for IBSR20 dataset 


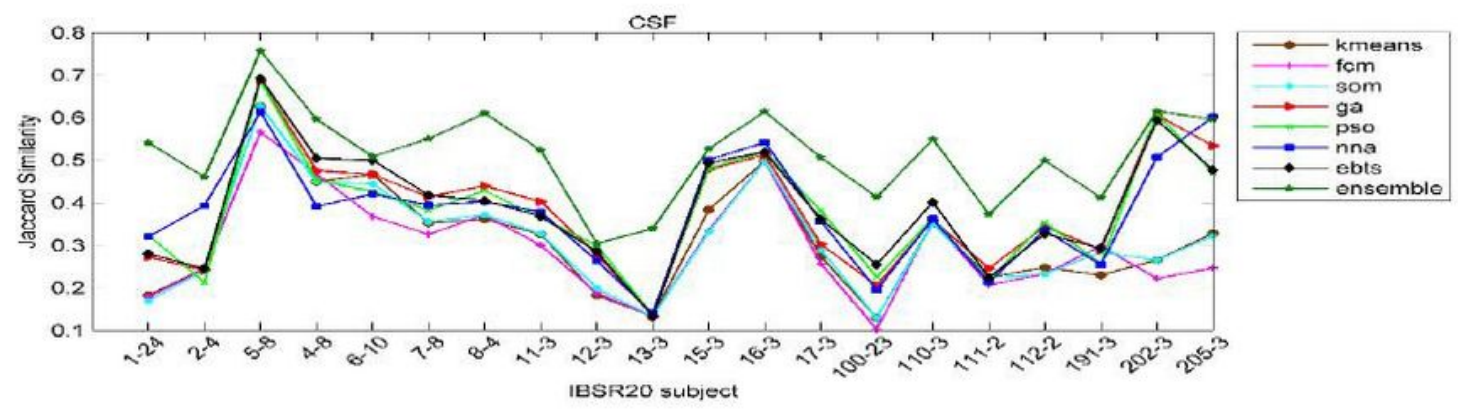

(a)

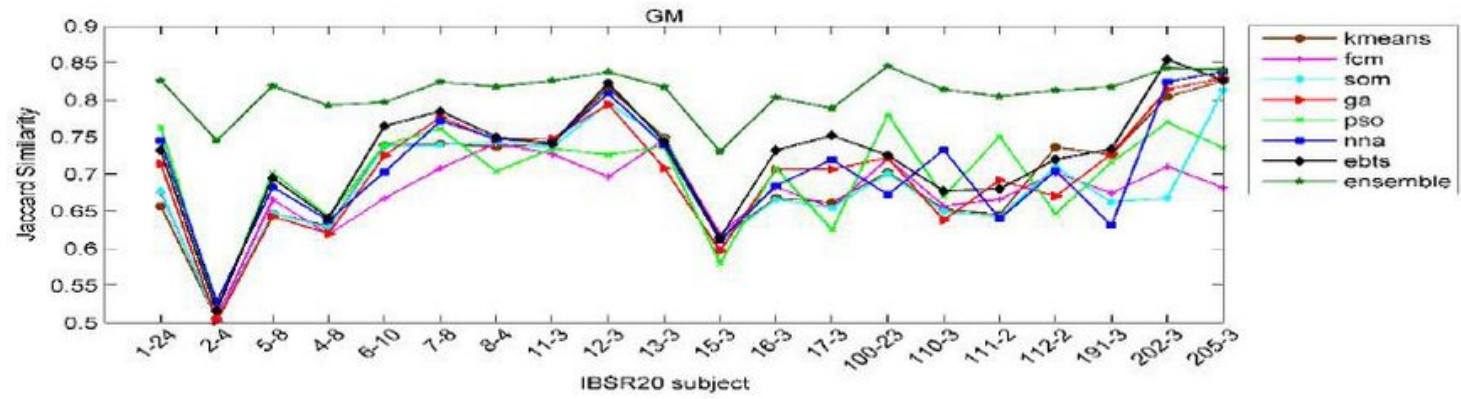

(b)

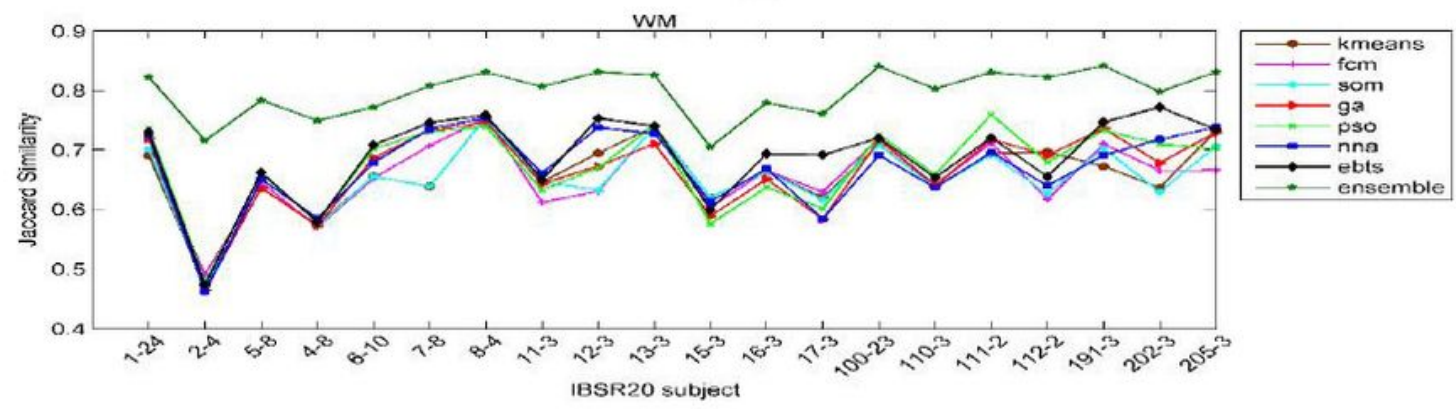

(c)

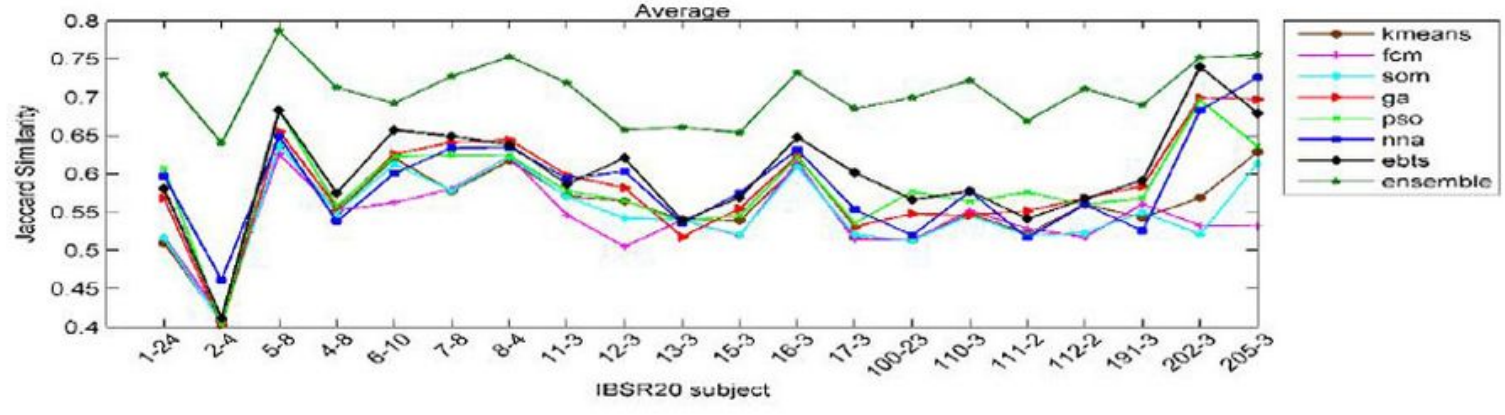

(d)

Figure 15

JS criterion results for IBSR20 dataset, slice 20: Error! Reference source not found. CSF, Error! Reference source not found. GM, Error! Reference source not found. WM, Error! Reference source not found. Average 